BNL-76859-2006-BC

\title{
Techniques for the Study of the Structural Properties
}

\author{
José A. Rodriguez ${ }^{1}$, Marcos Fernández-Garcia ${ }^{2}$, Arturo Martínez- \\ Arias $^{2}$, Jonathan C. Hanson ${ }^{1}$ \\ ${ }^{1}$ Brookhaven National Laboratory, Upton, NY 11973, USA \\ ${ }^{2}$ Instituto de Catálisis y Petroleoquímica CSIC, 28049 Madrid, SPAIN
}

To be published in "Synthesis, Properties and Applications of Oxide Nanomaterials"

June 2006

Chemistry Department
Brookhaven National Laboratory
P.O. Box 5000
Upton, NY 11973-5000
www.bnl.gov

\begin{abstract}
Notice: This manuscript has been authored by employees of Brookhaven Science Associates, LLC under Contract No. DE-AC02-98CH10886 with the U.S. Department of Energy. The publisher by accepting the manuscript for publication acknowledges that the United States Government retains a non-exclusive, paid-up, irrevocable, world-wide license to publish or reproduce the published form of this manuscript, or allow others to do so, for United States Government purposes.
\end{abstract}

This preprint is intended for publication in a journal or proceedings. Since changes may be made before publication, it may not be cited or reproduced without the author's permission. 


\section{DISCLAIMER}

This report was prepared as an account of work sponsored by an agency of the United States Government. Neither the United States Government nor any agency thereof, nor any of their employees, nor any of their contractors, subcontractors, or their employees, makes any warranty, express or implied, or assumes any legal liability or responsibility for the accuracy, completeness, or any third party's use or the results of such use of any information, apparatus, product, or process disclosed, or represents that its use would not infringe privately owned rights. Reference herein to any specific commercial product, process, or service by trade name, trademark, manufacturer, or otherwise, does not necessarily constitute or imply its endorsement, recommendation, or favoring by the United States Government or any agency thereof or its contractors or subcontractors. The views and opinions of authors expressed herein do not necessarily state or reflect those of the United States Government or any agency thereof. 


\title{
Chapter 5
}

\section{Techniques for the Study of the Structural Properties}

\author{
José A. Rodriguez \\ Chemistry Department \\ Brookhaven National Laboratory \\ Upton, NY 11973, USA \\ Marcos Fernández-García \\ Instituto de Catálisis y Petroleoquímica \\ CSIC, Campus Cantoblanco \\ 28049 Madrid, Spain \\ Arturo Martínez-Arias \\ Instituto de Catálisis y Petroleoquímica \\ CSIC, Campus Cantoblanco \\ 28049 Madrid, Spain \\ Jonathan C. Hanson \\ Chemistry Department \\ Brookhaven National Laboratory \\ Upton, NY 11973, USA
}

\section{Table of contents:}

5.1 Techniques for the study of structural properties

\subsection{XRD and PDF}

\subsection{XAFS}

\subsection{Raman spectroscopy}

\subsection{TEM and STEM}

5.6 STM and AFM

References 


\subsection{Techniques for the study of structural properties}

The evolution of our understanding of the behavior of oxide nanostructures depends heavily on the structural information obtained from a wide range of physical methods traditionally used in solid state physics, surface science and inorganic

chemistry. ${ }^{1,2}$ In this chapter, we describe several techniques that are useful for the characterization of the structural properties of oxide nanostructures: X-ray diffraction (XRD) and scattering, X-ray absorption fine structure (XAFS), Raman spectroscopy, transmission electron microscopy (TEM), scanning tunneling microscopy (STM) and atomic force microscopy (AFM). The ultimate goal is to obtain information about the spatial arrangement of atoms in the nanostructures with precise interatomic distances and bond angles. This may not be possible for complex systems and one may get only partial information about the local geometry or morphology.

\subsection{XRD and PDF}

Since the 1910s, X-ray crystallography has been a valuable tool for obtaining structural parameters of metal oxides. ${ }^{1} \mathrm{X}$-ray diffraction powder patterns come from the interference pattern of elastically dispersed X-ray beams by atom cores and, in the case of materials with moderate to long range order, contain information which arises from the atomic structure and the particle characteristics (for example: size, strain). ${ }^{1,2}$ The effect of atomic structure on peak positions and intensities is described in text books ${ }^{1}$ and the effect of particle characteristics on peak shape has been recently reviewed. ${ }^{3}$ This technique is frequently used for studying the structural properties of non-amorphous oxide nanoparticles. $^{2}$ 
Alternatively, the radial distribution of the materials can be determined by the appropriate Fourier transform of the diffraction pattern independent of any requirement of long range coherence. This is an advantage in the study of nanoparticles or nanostructures where there is reduced long range order. Egami and coworkers have applied this technique to metal oxide particles and obtained information about the structure and its defects. ${ }^{4}$ Finally, low angle x-ray diffraction ${ }^{5}$ can be used to determine the structure of nanoparticles.

Particle characteristics from whole powder profile fitting. Recent analyses of particle characteristics rely on least squares refinement of the whole powder diffraction profile, although there are several useful approximations which give some of these characteristics from individual peaks. The pattern is represented as a superposition of peaks where the peak position depends on the cell parameters, and the peak width is a sum of instrument parameters, particle size parameters $(\tau)$, and strain related parameters $(\varepsilon)$. The particle size peak width is given by

$$
\beta_{\tau}=\mathrm{K} \lambda / \tau \cos \theta
$$

while the strain peak width varies as

$$
\beta_{\varepsilon}=4 \varepsilon \tan \theta
$$

with $\lambda$ representing the wavelength and $\theta$ the diffraction angle. The fact that the strain broadening follows a $\tan \theta$ function whereas the crystallite size broadening has a $1 / \cos \theta$ dependence allows the separation of these effects in whole profile fitting through a range of $\theta .{ }^{6}$ Here, the strain $(\varepsilon)$ can be the result of a non-uniform application of stress in the lattice of the nanoparticles and reflects variations in cell dimension within the sample. Obvious variations are present at the surface of the material with respect to bulk since 
local symmetry and distances are different. Additionally, Oxygen vacancies and the different types of defects ${ }^{7}$ shown in Table 1 can lead to strain in the lattice of a nanoparticle. The relative importance of each type of defect depends on the size of the nanoparticle and the chemical nature of the oxide.

Additional structural features can be obtained from diffraction. In some cases, the refinement of the powder pattern can include structural parameters for the thermal motion of the atoms, the fractional occupancy and the positions. The quality of these refinements can be greatly improved with high energy X-rays were the absorption correction is negligible and a larger range of $\mathrm{Q}(4 \pi \sin (\theta) / \lambda)$ can be measured. ${ }^{8}$

Local structure from Fourier analysis of whole pattern. The local structure from amorphous nanoparticles can be determined from the diffraction pattern in a manner similar to techniques which have been used to determine the structures of liquids and glasses. ${ }^{9}$ As an example, we describe the determination of the atomic pair distribution function (PDF) of amorphous quartz. Figure 1a shows the diffraction pattern from a quartz capillary. The local structure $\mathrm{g}(\mathrm{r})$ is defined as the probability of finding an atom at a distance $\mathrm{r}$ from a reference atom and is derived from $\mathrm{G}(\mathrm{r})$.

$$
\mathrm{G}(\mathrm{r})=4 \pi \mathrm{r}\left[\rho(\mathrm{r})-\rho_{0}\right] \quad \rho(\mathrm{r})=\rho_{0} \mathrm{~g}(\mathrm{r})
$$

Where $\rho(r)$ is the pair density function and $\rho_{0}$ is the average pair density. $\mathrm{G}(\mathrm{r})$ is also the Fourier transform of the total structure factor, $\mathrm{S}(\mathrm{Q})$

$$
\begin{aligned}
& \mathrm{G}(\mathrm{r})=(2 / \pi) \int \mathrm{Q}[\mathrm{S}(\mathrm{Q})-1] \sin (\mathrm{Qr}) \mathrm{dQ} \\
& \mathrm{Q}=4 \pi \sin (\theta) / \lambda
\end{aligned}
$$

The structure factor $\mathrm{S}(\mathrm{Q})$ is related to elastic part of the diffraction intensity $\left(\mathrm{I}^{\mathrm{el}}\right)$ as follows: 


$$
\mathrm{S}(\mathrm{Q})=1+\left[\mathrm{I}^{\mathrm{el}}(\mathrm{Q})-\sum \mathrm{c}_{\mathrm{i}}\left|\mathrm{f}_{\mathrm{i}}(\mathrm{Q})\right|^{2}\right] /\left|\sum \mathrm{c}_{\mathrm{i}} \mathrm{f}_{\mathrm{i}}(\mathrm{Q})\right|^{2}
$$

where $c_{i}$ is the fraction of species $i$, and $f_{i}(Q)$ is the atomic scattering factor of species $i$. The $\mathrm{I}^{\mathrm{el}}$ is obtained from the total scattering after subtraction of background, and corrections for Compton scattering, absorption, multiple scattering. The calculated $G(r)$ is shown in figure $1 \mathrm{~b}$. In this figure we clearly see the $\mathrm{Si}-\mathrm{O}, \mathrm{O}-\mathrm{O}$ and $\mathrm{Si}-\mathrm{Si}$ distances and some longer distances. The number of inter-atomic distances has decreased to nearly zero by the distance of $10 \AA$.

The real space resolution obtained in the PDF is directly dependent on the Q range measured and it is therefore a necessity to measure data to high $\mathrm{Q}$, with adequate counting statistics, to obtain well resolved peaks in the PDF. ${ }^{10}$ Generally the data are collected at synchrotrons where high energy (short wavelength) and high intensity facilitate the above requirement. When the PDF technique is applied to crystalline materials, it has the advantage that the diffuse scattering data in the "background"(not under Bragg diffraction peaks) region contributes to the PDF. The obvious disadvantage of having a one dimensional result is partly compensated for by refining threedimensional models of the one dimensional data. ${ }^{11}$

The PDF analysis of nanoparticles of ceria is another good example of the application of this tool to nanostructures of oxides. ${ }^{12}$ In this case, data of neutron diffraction was used in the analysis. The neutron scattering cross sections from cerium and oxygen are nearly equal while X-ray diffraction scattering by oxygen is much less than cerium and, consequently, neutron diffraction provides a more reliable determination of the oxygen structural features. However, a recent experiment on nano ceria using high energy X-rays $(\lambda=0.15)$ obtained similar results. ${ }^{13}$ Frenkel defects of the oxygen atoms in 
ceria were observed using this technique. Recent PDF studies ${ }^{14}$ of $\mathrm{Zr}$ doped ceria have show that the oxygen storage capacity can be related to interfacial domains between the zirconium and cerium rich domains.

In addition to nano $\mathrm{CeO}_{2}$ and $\mathrm{Ce}_{1-\mathrm{x}} \mathrm{Zr}_{\mathrm{x}} \mathrm{O}_{2}$, several structures of nano-crystalline metal oxides have been elucidated using the PDF technique. ${ }^{15-17}$ In these systems the Bragg peaks were very broad and there was very limited long range order. The short range structures were solved by matching the observed $G(R)$ to related bulk structures. Nano tubes of of $\mathrm{V}_{2} \mathrm{O}_{5}$ have a very well defined structure based on double layers of pyramidal/octahedral vanadium-oxygen units derived from the structure of $\mathrm{BaV}_{7} \mathrm{O}_{16} \cdot \mathrm{nH}_{2} \mathrm{O} .{ }^{15}$ In a similar way, the structure of a $\mathrm{V}_{2} \mathrm{O}_{5}-\mathrm{nH}_{2} \mathrm{O}$ Xerogel was shown to consist of square pyramidal $\mathrm{V}_{2} \mathrm{O}_{5}$ at the nanometer length scale. ${ }^{16}$ In addition, the structure of hydrated lithium manganese oxide was found to contain layers made up of edge shared $\mathrm{MnO}_{6}$ octahedra encapsulating $\mathrm{K}$ and $\mathrm{Li}$ atoms with the hydrous sample and $\mathrm{Li}$ and $\mathrm{Mn}$ atoms within the anhydrous sample. ${ }^{17}$ This result indicates that the improved electrochemical performance of the water based nano-crystalline lithium manganese oxide could be attributed to its unique structure with well separated MnO layers leaving Li atoms ample room to move in and out during the charge-discharge process. ${ }^{17}$

In situ time-resolved powder diffraction. Investigations at Brookhaven National Laboratory and other institutions have established the feasibility of conducting subminute, time-resolved X-ray diffraction (XRD) experiments under a wide variety of temperature and pressure conditions $\left(-190{ }^{\circ} \mathrm{C}<\mathrm{T}<900{ }^{\circ} \mathrm{C} ; \mathrm{P}<45 \mathrm{~atm}\right) .{ }^{18,19,20}$ This is made possible by the combination of synchrotron radiation with new parallel data- 
collection devices. Using time-resolved XRD one can follow structural changes during the preparation of nanoparticles and under reaction conditions. Figure 2 shows timeresolved powder diffraction results for $\mathrm{Zr}$ doped nano ceria $\left(\mathrm{Ce}_{0.9} \mathrm{Zr}_{0.1} \mathrm{O}_{2}\right)$ during heating from 25 to $925^{\circ} \mathrm{C}$ in air. ${ }^{21}$ The sintering of the nanoparticles produces a sharpening of the diffraction peaks at temperatures above $600{ }^{\circ} \mathrm{C}$. This type of approach can also provide information of changes in cell dimension or lattice strain with temperature.

Recently, the PDF technique has been extended/modified to allow the rapid acquisition of the necessary data. Time-resolved PDF measurements of nano catalysts under reaction conditions are now in progress. ${ }^{22}$

\subsection{XAFS}

The X-ray absorption fine structure (XAFS) acronym refers to the oscillatory structure observed in the absorption coefficient $(\mu(\mathrm{E}))$ just above or close to the absorption edge of an element constituting the sample. X-rays absorption spectroscopies are, essentially, synchrotron based techniques and measure the absorption coefficient as a function of the X-ray energy $E=h \omega$. A typical absorption spectra $(\mu(E)$ vs. E) show three general features. The first one is an overall decrease in $\mu(E)$ with increasing energy which, sometimes, also contains well defined peaks at specific energies called pre-edge transitions. The second is the presence of the edge, which roughly resembles a step function with a sharp rise at the corresponding edge energy and reflects the ionization of an inner-shell (or core) electron of the element under study. The third component appears above the edge and corresponds to the oscillatory structure that can be roughly described as a periodical function, progressively damped as evolves from the edge energy. Being 
absorption techniques, XAFS spectroscopies are element-specific. On the other hand, the deep penetration of X-rays in the matter makes them to probe the whole system, being only surface sensitive by performing specific detection schemes will be briefly discussed below.

The XAFS spectrum, $\chi(\mathrm{E})$, is defined phenomenologically as the normalized oscillatory structure of the X-ray absorption, e.g. $\chi(E)=\left(\mu(E)-\mu_{0}(E) / \mu_{0}(E)\right)$, where $\mu_{0}(E)$ is the smooth varying atomic-like background absorption. Essentially, the XAFS spectrum involves the quantum-mechanical transition from an inner, atomic-like core orbital electron to unoccupied bound (pre-edge transition) or unbound, free-like continuum levels. The oscillatory structure therefore reflects the unoccupied part of the electronic bands/structure of the system in presence of a core-hole ${ }^{23,24,25,26,27}$. Note that this differs from the initial, ground state by physical effects induced by the fact that the core-hole is not infinitely long lived but must decay as a function of time and distance from the photoabsorber atom ${ }^{20-24}$. The latter is a point to stress here as their short range order character makes these techniques particularly suitable to analyze nanostructured materials which, frequently, do not posses or have a strongly disturbed long range order.

Historically, it was controversial to recognize the local nature of the XAFS techniques (see Lytle for a recent discussion ${ }^{28}$ ) but now the theoretical description of the techniques is reasonably well settled ${ }^{22,23}$. The absorption of X-rays by matter is described in many text books ${ }^{29}$. The treatment of the radiation as an electric field without practical spatial variation on a molecular/local scale and eliminating magnetic parts leads to the Fermi Golden Rule for the X-ray cross section:

$$
\mu(E)=K\left[\left\langle\phi_{f} / e \cdot \bar{r} / \phi_{i}\right)\right]^{2} \delta_{E f-E i+h \gamma}
$$


The intensity of the absorption process is then proportional to the square of the transition matrix element connecting the initial $\left(\phi_{\mathrm{i}}\right)$ and final $\left(\phi_{\mathrm{f}}\right)$ states times a delta function which ensures the fulfillment of the conservation energy theorem. The elimination of the spatial dependence of the electric field corresponds to a series expansion of its $\mathrm{e}^{2 \pi z / \lambda}$ dependence up to the first term (linear dependence in $\mathrm{r}$-equation 5.3.1); this yields the dipole approximation of the interaction energy between the atom electronic cloud and the X-ray radiation field. Better approximations will include quadrupole, octupole and so forth terms. However, except in few cases, some of them here detailed, the dipole approximation gives quantitative analysis of the XANES shape.

An important approximation is to assume that the matrix element can be rewritten into a single-electron matrix element. This is based in the sudden approximation which allows to reorder the transition element in 5.3.1 in terms of a overlap term of the N-1 "inactive" electrons, which is roughly independent of energy, and the mentioned singleelectron matrix element connecting wavefunctions of the unbound and inner-core electrons ${ }^{30}$. The validity of the sudden approximation depends primarily on the photoexcited electron kinetic energy and has been the subject of many studies ${ }^{21,25,27}$. All electron rearrangements (inter-atomic and mainly extra-atomic relaxation of valence electrons) in response to the core-hole creation are thus neglected, the series of delta functions corresponding to different final states identifies with the local density of states $(\rho)^{31}$ and the corresponding X-ray absorption cross section becomes: 


$$
\mu(E)=K\left[\left\langle\varphi_{f} / e \cdot \bar{r} / \varphi_{i}\right)\right]^{2} \rho
$$

Where $\varphi_{\mathrm{i}, \mathrm{f}}$ correspond to the mentioned initial and final mono-electronic wavefunctions. The dipole matrix element dictates that the local density of states has an orbital moment that differs by 1 from the core state $(\Delta \mathrm{L}= \pm 1)$ while the spin is conserved $(\Delta \mathrm{S}=0)$. If the electric quadrupole radiation is considered, the selection rules change and are $(\Delta \mathrm{L}=0, \pm 2$; $\Delta \mathrm{S}=0 ; \pm 1, \pm 2)$.

There are two XAFS techniques; X-ray absorption near edge spectroscopy (XANES) and extended X-ray absorption fine structure (EXAFS). They differ in the energy of the final electronic state sampled which is limited to a maximum of about 40$50 \mathrm{eV}$ for XANES and above that point for EXAFS (Figure 3). Intuitively, it is obvious that pure electronic information (e.g. chemical bonding information) is only enclosed in the low-lying extended states and is thus confined to the XANES region. This will be discussed in Chpt. 6 of this book. On the other hand, at high energy in the continuum of electrons participating in EXAFS, the effect of neighboring atoms becomes small and electron states approximate to spherical waves that are simply scattered by such atoms. The information extracted is thus of geometrical local character.

As a general rule, equations 5.3.1 and 5.3.2 can be solved by using modern quatum-mechanical methods. Two general approaches are discerned. The first one employs Ab-initio or DFT methods to calculated the initial and final wavefunctions of the electronic transition. The second reformulates the Schorodinger equation in terms of the scaterring theory and allows to express the absorption equation as a correlation function. 
This latter is particularly useful for EXAFS analysis of nanostructured materials as they can be treated as clusters for calculations which, in turn, can be performed in steps of growing size, showing the effect of introducing different coordination spheres. Detailed accounts for the multiple scattering EXAFS theory can be found in refs. 32, 33. Using this theoretical framework the EXAFS formulae can be written as:

$$
\chi(E)=S_{0}^{2} \sum_{i} \frac{N_{i} F(k)}{k R_{i}^{2}} e^{-2 R / \lambda(k)} e^{-2 D W} \sin (2 k R+\phi(k))
$$

This was originally proposed by Sayer et $a .^{34}$, based in a single-scattering formulism but can be generalized to represent the contribution of $\mathrm{N}$ equivalent multiplescattering contributions of path length $2 \mathrm{R}^{31}$. It can be thus considered the standard EXAFS formulae, providing a convenient parameterization for fitting the local structure around the absorbing atoms. The dependence of the oscillatory structure on interatomic distance and energy is reflected in the $\sin (2 \mathrm{kR})$ term. The decay of the wave due to mean free path or lifetime (including intrinsic core-hole and extrinsic inelastic losses) is enclosed in the exponential $2 \mathrm{R} / \lambda$, which is largely responsible for the relatively short range probed by EXAFS in a material. As early recognized in ref. 32, the sinusoidal nature of the EXAFS phenomenon allows a Fourier analysis of the signal, yielding key information to give initial guesses for the fitting parameters. In fact, for each shell contributing to the EXAFS signal we may have a peak in the Fourier Transform spectrum. The strength of the interfering waves depends on the type and number $(\mathrm{N})$ of neighboring atoms through the backscattering amplitude $(\mathrm{F}(\mathrm{k}))$ which largely dictates the magnitude of the signal. The phase function $(\phi(\mathrm{k}))$ reflects the quantum-mechanical 
wavelike nature of backscattering which depends on both the absorber and the scatterer atom properties and accounts for the difference between the measured and geometrical interatomic distances (which is typically of 0.3-0.4 A for low atomic number (Z) bacscatterers and lower for the rest of atoms) displayed in the Fourier Transform ${ }^{24,26}$.

Another important factor is the exponential Debye-Waller (DW) term. This accounts for thermal and static disorder effects concerning the movement/position of atoms around their equilibrium/averaged position. A point to stress is that the nature of this term is different to the counterpart term in $\mathrm{XRD}^{35}$. Since vibrations increase with temperature, EXAFS spectra are usually acquired at low temperature in order to maximize information. Spectra at different temperatures may, on the other hand, allow to decouple thermal and static contributions to DW. The DW term smears the sharp interference pattern of the sinusoidal term and cut off EXAFS at sufficiently large energy beyond ca. $20 \mathrm{~A}^{-1}$. Important to stress is that the DW factor is in fact a complex mathematical function and has a natural cumulant expansion in powers of $\mathrm{k}$. The amplitude of Debye-Waller term contains even moments $\mathrm{DW}(\mathrm{k})=2 \mathrm{C}_{2} \mathrm{k}^{2}-(2 / 3) \mathrm{C}_{4} \mathrm{k}^{4}+\ldots$ while odd moments contribute to the EXAFS phase $\phi(k)=2 c_{1} k-(4 / 3) C_{3} k^{3}+\ldots{ }^{36}$. While the fitting of bulk systems makes use up to the squared term of the k-series expansion, the point is that the $\mathrm{k}^{3}$ behavior (third cumulant) is important for "disordered" systems; as surface atoms have a less symmetric environment with respect to bulk ones and correspond to a significant part in nanostructured materials, the cumulant expansion need to be considered in analyzing EXAFS data. Inclusion of the third cumulant would mainly influence coordination distance while the fourth would influence coordination number and/or (second order) Debye-Waller factor. 
Effect of Nanostructure. EXAFS analysis of oxides as a function of size are scarce and mainly devoted to study $\mathrm{Ti}^{37,38,39}, \mathrm{Zn}^{40} \mathrm{Y}^{41}, \mathrm{Zr}^{41,42}$ and $\mathrm{Ce}^{43,44,45}$ containing systems. A point to stress is that, as mentioned throughout the text, nanocrystalline phases prepared by chemical methods are commonly accompaigned by amorphous ones ${ }^{43,46}$ and different techniques may in fact be selective to one of them (XRD) while others (as XAFS) inform over the whole system. Although EXAFS studies of dispersed surface species are not the main target of this review, it can be mentioned here that revisions of related papers can be found in refs. 47 and 48 . Surface species can have isolated or oligomeric structures, differing in their local order from the surface termination of nanoparticulated materials by effect of the oxide anion sharing between support and surface components. In this case, there are an infinite number of possible local arrangements as a function of the support nature, oxides like alumina, silica, or carbon-containing materials (surface-modified carbon using oxygen-containing groups, nanotubes, etc.) and morphological properties (surface area and face exposed to the atmosphere) and the loading of the surface species.

Analysis of the EXAFS data of nanostructured oxides which account for the relative asymmetric (anharmonic) pair distribution of bond distances are even more scarce and exclusively devoted to $\mathrm{Ti}$ and Ce oxide systems ${ }^{49,50}$. This however has been frequently worked out for nanostructured chalcogenides ${ }^{51}$ and has been studied in detail for nanostructured fcc metals ${ }^{52}$. As metal oxides display multitude of different crystal structures, no general correlation between EXAFS coordination numbers of the three or four first shell coordination numbers and average particle size/morphology has been published. This can be found for fcc metals ${ }^{53}$ and might be generalized to certain oxide 
cubic structures. It can be however noted that first shells display coordination numbers (CN) rather close to bulk ones, differing typically by less than one unit as undercoordinated atoms complete their first coordination shell at surfaces by using hydroxyl groups (see below). Second and further shells display CNs departing from bulk values but their analysis is structure and shape dependent. A point to mention is that in some case, like $\mathrm{ZrO}_{2}$ oxides, coexistence of two crystalline (or one amorphous) phases can lead to destructive interference effects and misleading interpretation of results. ${ }^{54}$

For titanium oxides, the studies usually identify the presence of fivefoldcoordinated species in the nanostructured anatase and rutile surfaces, with a shortening of the Ti-O first shell bond distance ${ }^{37,38}$ which in some cases is identified as a result of presence of Ti hydroxyl groups at the surface ${ }^{37}$. Note however that the inclusion of the third cumulant for studying mesoporous (amorphous by XRD but with a surface local structure similar to nanostructured anatase) titania suggests a larger Ti-O average first coordination distance with respect to the rutile/anatase phase ${ }^{50}$. The presence of surface hydroxyl-coordinated centers has been also mentioned in $\mathrm{ZrO}_{2}$ but detected by using other techniques ${ }^{42}$. The case of cerium-containing nanostructured oxides is still under study due the fact that the series of samples with increasing size are obtained by calcining at progressively higher temperatures solids from Ce(III) precursors and this influences the presence and concentration of two oxidation states $\mathrm{Ce}(\mathrm{III}) / \mathrm{Ce}(\mathrm{IV})$ in materials with small particle size $\mathrm{s}^{43,44}$.

Geometrical information is also enclosed in the XANES spectrum. The energy position of the peaks corresponding to electronic transitions to final quasibound, continuum states (called continuum resonances, CRs, in solid state physics) depends on 
both electronic and geometrical factors ${ }^{23,29,55}$. Electronic factors are analyzed in chapter 6. Geometrical factors are summarized in the so-called $1 / \mathrm{R}^{2}$ rule, which states that the quantity $\Delta \mathrm{E} * \mathrm{R}^{2}$ is a constant, where $\Delta \mathrm{E}$ refers to the energy difference between the $\mathrm{CR}$ energy position and the zero electron kinetic energy and $\mathrm{R}$ is the nearest neighbor distance. This rule has been used fruitfully in solid-state studies but an article by Kizler ${ }^{56}$ demonstrates limitations in its applicability. Essentially, this rule can be used with generality only for CRs dominated by scattering from first or second nearest neighbors.

\subsection{Raman Spectroscopy}

Raman spectroscopy is a powerful tool to analyze structural/morphological properties of solid oxides at a local level, given the strong sensitivity of the phonon characteristics to the crystalline nature of the materials. ${ }^{57}$ The theoretical basis for the application of this technique to the study of the properties of extended crystals as well as of individual molecular complexes present at the surface of oxide materials is out of the scope of the present work and can be found in different textbooks or review articles. ${ }^{58,59}$ Raman spectroscopy is a bulk sensitive technique but the use of excitation frequencies allowing charge-transfer band absorption (Resonance Raman) allows some surface sensitivity. Essentially, the theoretical background for the study of nanocrystalline (considered as a whole as crystalline materials) oxides as well as the spectral consequences observed by Raman is provided by the phonon confinement model that will be briefly analyzed in a first part; complete details of this model can be found elsewhere. ${ }^{60,61,62}$ The study by Raman spectroscopy of other properties that can in some cases be linked to the nanoscopic nature of the oxide materials, like the existence of 
disorder associated to the presence of lattice defects (see also section 5.1) or surface phonons, will be described later.

Phonon confinement model. The model employed for analyzing the features observed in the Raman spectra of nanocrystalline materials is the so called "phonon confinement" (or "spatial correlation") model. ${ }^{63}$ Within such model, nanocrystalline materials can be considered as an intermediate case between that of a perfect infinite crystal and that of an amorphous material. Thus, in a perfect crystal, conservation of phonon momentum $(q)$ during phonon creation or decay upon interaction with the external radiation field requires that, in first-order Raman scattering, only optic phonons near the center of the Brillouin zone $(q \approx 0)$ are involved. In amorphous materials, due to the lack of long-range order, the $q$ vector selection rule does not apply and the Raman spectrum resembles the phonon density of states. In the intermediate case of nanocrystalline materials, due to the uncertainty principle, a range of $q$ vectors of the order $\Delta q \approx 1 / \mathrm{L}$ (where $\mathrm{L}$ is the particle size) can be involved in the Raman process. ${ }^{60-63}$ The approach employed in the phonon confinement model follows from the work by Richter et al., ${ }^{60}$ which shows that the relaxation of the conservation of phonon momentum selection rule arises from substitution of the phonon wave function in an infinite crystal by a wave function in which the phonon is confined in the volume of the crystallite. In their approach, which is employed to explain the first-order Raman spectrum of microcrystalline spherical silicon particles, it is used a Gaussian function to impose the phonon localization. Later, Campbell and Fauchet extend the model by using other confinement functions and considering different shapes for the nanocrystallites (spherical, columnar or thin films). ${ }^{62}$ They found that the Raman spectrum of different 
semiconductor nanocrystals is best explained by using a Gaussian confinement function similar to the one employed by Richter et al., although with lower amplitude at the boundaries. By using this model, the first order Raman spectrum is, considering the general case of a Gaussian confinement function with phonon amplitudes at the borders depending on a confinement parameter $\beta,^{60-63}$ given by

$$
I(\omega) \cong \int_{B Z} \frac{d^{3} q \exp \left(-q^{2} L^{2} / 8 \beta\right)}{(\omega-\omega(q))^{2}+\left(\Gamma_{0} / 2\right)^{2}}
$$

where the integration is carried out over the entire Brillouin zone in the corresponding symmetry directions, $\omega(q)$ is the phonon dispersion curve and $\Gamma_{0}$ is the natural linewidth. In the case of an infinite crystal $(\mathrm{L} \rightarrow \infty), \mathrm{I}(\omega)$ is a Lorentzian centered at $\omega(0)$ (the Raman frequency) with a linewidth of $\Gamma_{0}$. Application of equation (5.4.1) to any given Raman line of the perfect crystal results, as experimentally observed, ${ }^{60-64}$ in frequency shifts and asymmetric broadenings that increase with decreasing the particle size of the nanocrystal, and of a sign and magnitude that depends on the form and dispersion of the $\omega(q)$ function. Thus, a negative/positive dispersion in $\omega$ values as $q$ increases is expected to produce asymmetric low/high frequency broadening and a red/blue shift of the peak.

Examples on the application of the phonon confinement model to the analysis of Raman lines in nanosized $\mathrm{TiO}_{2}$ and $\mathrm{SnO}_{2}$ materials ${ }^{60,65}$ are shown in Fig. 4; similar analyses successfully applied to the study of Raman lines in $\mathrm{ZrO}_{2}$ or $\mathrm{ZnO}$ nanoparticles are found in the literature. ${ }^{65,66}$ In the general case, a qualitative approach to the model is used to explain the evolutions of Raman peaks with decreasing the size of the nanoparticles. In this sense, it must be considered that in addition to the uncertainty in the choice of the confinement function and phonon dispersion curves (in the absence of 
precise theoretical or experimental results by neutron inelastic scattering), the presence of non-uniform particle size distributions or of imperfect crystallites (further confining the phonons by the microdomain boundaries or defects) often leads to resort to empirical correlations for the analysis. ${ }^{64,65,66,67,68,69,70,71,72}$ Some examples of application of the confinement model for qualitative interpretation of Raman results in series of nanostructured oxides like anatase $\mathrm{TiO}_{2}, \mathrm{CuO}, \mathrm{Cr}_{2} \mathrm{O}_{3}, \mathrm{ZrTiO}_{4}$ or manganese oxides can be found elsewhere. ${ }^{67-73}$

In some cases, refinement of the model by including effects of defects or more precise consideration of particle size distributions leads to closer approaches to the experimental results, as shown by Spanier et al. for $\mathrm{CeO}_{2}$ nanoparticles. ${ }^{63}$ Thus, the analysis of the three-fold degenerated $F_{2 g}$ peak at $465 \mathrm{~cm}^{-1}$ (the only one allowed in first order) in $\mathrm{CeO}_{2}$ nanosized samples with multidisperse size distributions requires consideration of both the effects of the presence of the vacancies (proposed to increase with decreasing particle size, at least for sizes below ca. $2 \mathrm{~nm})^{74}$ and the associated change in lattice dimensions. While the former, according to experimental evidence, is expected to give rise to blue shifts of the peak, this is compensated by the red shift produced upon lattice expansion $(\Delta \mathrm{a})$, in accordance with the equation:

$$
\Delta \omega=-3 \gamma \omega_{0}\left(\Delta \mathrm{a} / \mathrm{a}_{0}\right)
$$

where $\omega_{0}$ is the $\mathrm{F}_{2 \mathrm{~g}}$ frequency in pure stoichiometric $\mathrm{CeO}_{2}, \mathrm{a}_{0}$ is the $\mathrm{CeO}_{2}$ lattice constant, and $\gamma=(\mathrm{B} / \omega) \mathrm{d} \omega / \mathrm{dP}$ is the Grüneisen constant with $\mathrm{B}$ the bulk modulus and $\mathrm{d} \omega / \mathrm{dP}$ the linear shift of the frequency with hydrostatic pressure. ${ }^{75}$ The result is that the red shift and broadening observed in the Raman line is significantly larger than expected from the phonon confinement model and adequate fitting of the experimental spectra is only 
obtained when jointly considering the effects of inhomogeneous strain (associated to the dispersion in lattice constants as a consequence of the presence of vacancies and reduced cerium centers) ${ }^{74}$ and phonon confinement. ${ }^{63}$ A similar approach has been employed to explain qualitatively the shifts observed in the main band of $\mathrm{Ce}-\mathrm{Tb}$ or $\mathrm{Ce}-\mathrm{Cu}$ mixed oxide nanoparticles as a function of the $\mathrm{Tb}$ or $\mathrm{Cu}$ content. ${ }^{76,77}$ Another effect of the presence of oxygen vacancies in the $\mathrm{CeO}_{2}$ nanoparticles is the appearance of a new broad peak at ca. $600 \mathrm{~cm}^{-1}$ that can be related to a second-order phonon or a local mode centered on the vacancy positions. ${ }^{63,75,78}$ The presence of second order peaks associated to nanostructured configurations has been also reported for $\mathrm{ZrO}_{2}{ }^{79}$

Observation of acoustic modes. Another possible consequence of decreasing the particle size in the nanosized oxides is the appearance of low frequency bands associated to acoustic modes. An example is the study performed on $\mathrm{SnO}_{2}$ which, for particles from 3.7 to $9.5 \mathrm{~nm}$ of average size, displays a peak from ca. 15 to $60 \mathrm{~cm}^{-1}$ that shifts to higher frequency and becomes less intense and broader with decreasing the particle size (Fig. 5 shows the spectrum obtained under typical backscattering geometry of the spectrometer; note that correction of Bose-Einstein phonon occupation factor required for the analysis is not included). ${ }^{80}$ Analysis of such peak considers the vibration of the nanoparticle as a whole. Thus, the nanoparticle is considered as an elastic body of spherical shape with two types of vibrational modes, spheroidal and torsional. Then, after considering different boundary conditions (rigid or stress free), one arrives to expressions for estimating the frequency of the vibration as a function of the particle size of the type: ${ }^{80}$

$$
\omega=\frac{S_{l, t} v_{l, t}}{L c}
$$


where $\mathrm{v}_{\mathrm{l}, \mathrm{t}}$ is one of the two sound velocities ( $\mathrm{v}_{\mathrm{l}}$ for spherical or longitudinal and $\mathrm{v}_{\mathrm{t}}$ for torsional or transversal vibrations) in the bulk of the oxide, $\mathrm{S}_{\mathrm{l}, \mathrm{t}}$ is a coefficient that depends on the sound velocities and $\mathrm{c}$ is the vacuum light velocity. As an interesting application of this analysis, non-destructive estimation of the particle size distribution can be done with this model after selecting the most adequate theoretical condition and applying it to deconvolute the experimental spectrum. ${ }^{80}$ Similar analyses have been applied to explain the presence of acoustical phonon modes in $\mathrm{ZrO}_{2}$ nanoparticles. ${ }^{81}$

Other spectral consequences of nanostructure. An obvious consequence of decreasing the particle size is that a relatively large fraction of atoms in the nanoparticle reside on the surface and, as a result, surface optical phonons can also be observed with measurable intensity and analyzed by Raman spectroscopy (in addition to high resolution electron energy loss spectroscopy and helium atom scattering techniques, most typically used for this purpose). An interesting application for this purpose is the use of resonant Raman spectroscopy tuned to the surface region, taking advantage of the different band structures of bulk and surface. ${ }^{82}$ The appearance of new vibrations (ascribed to surface phonon modes) different than those observed for the bulk material and increasing their relative intensity with decreasing the particle size has been observed in different oxide systems. ${ }^{80,83}$ A classical model is usually employed to analyze such surface phonons. According to the classical optical dispersion theory, each vibrational motion is characterized by an independent harmonic electric dipole oscillator with the corresponding transverse optical (TO) and longitudinal optical (LO) phonon modes. The frequency corresponding to the surface phonon modes must lie between $v_{\mathrm{TO}}$ and $v_{\mathrm{LO}}$ of any determinate TO-LO pair and satisfies the following condition: 


$$
v^{2}=v_{\text {то }}^{2}\left[\frac{\varepsilon_{0}+\varepsilon_{m}\left(1 / L_{k}-1\right)}{\varepsilon_{\infty}+\varepsilon_{m}\left(1 / L_{k}-1\right)}\right]
$$

where $\varepsilon_{0}, \varepsilon_{\infty}$ and $\varepsilon_{\mathrm{m}}$ are dielectric constants for the material (static and high-frequency ones) and the surrounding medium and $\mathrm{L}_{\mathrm{k}}$ are depolarization factors that depend on the particle shape. Full details of this theoretical approach and its successful application to the analysis of $\mathrm{SiO}_{2}$ surface modes can be found elsewhere. ${ }^{84}$

\subsection{TEM and STEM}

Transmission electron microscopy (TEM) is one of the most powerful and versatile techniques for the characterization of nanostructured systems. Its unique characteristics allow to achieve atomic resolution of crystal lattices as well as to obtain (with the assistance of energy dispersive X-ray spectroscopy - EDS - and electron energy-loss spectroscopy - EELS - complementary techniques) chemical and electronic information at the sub-nanometer scale. Numerous monographs have addressed technical or theoretical aspects involved in modern TEM microscopy employed for characterization of nanostructured systems as well as particular applications in different fields (see, for instance, refs. 85,86,87,88,89,90,91,92,93,94 and references therein). The vast extension of the field precludes giving an exhaustive treatment here and only a brief outline of fundamental aspects of the TEM application to characterization of nanostructured systems will be thus attempted. As it is well known, the interaction of an electron beam with a solid specimen results in a number of elastic or inelastic scattering phenomena (back-scattering or reflection, emission of secondary electrons, X-rays or optical photons and transmission of the undeviated beam along with beams deviated as a 
consequence of elastic - single atom scattering, diffraction - or inelastic phenomena). The TEM technique is dedicated to the analysis of the transmitted or forward-scattered beam. Such beam is passed through a series of lenses, among which the objective lens mainly determines the image resolution, in order to obtain the magnified image. In lowresolution TEM, the objective aperture will be adjusted for selection of the central beam (containing the less scattered electrons) or of a particular diffracted (or scattered in any form) beam to form the bright-field or dark-field image, respectively. In high-resolution TEM (HRTEM), which is usually performed in bright-field mode, the image is formed by collecting a few diffracted beams in addition to the central one.

Analysis of contrasts observed in the experimental TEM images when using the central beam by intercepting the electrons scattered through angles larger than a selected objective aperture is usually done on the basis of amplitude (or scattering) and massthickness or atomic number contrast theories ${ }^{85,86}$ Figure 6 shows a typical example of a bright-field image collected in this mode. ${ }^{95,96}$ The higher contrast resolution usually attained in dark-field with respect to bright-field images can be most useful to analyze particle size or shape of the nanoparticles; an interesting example in this sense has been shown in a work by Yin and Wang in which the tetrahedral shape of CoO nanoparticles is assessed from contrast analysis in dark-field images. ${ }^{97}$ On the other hand, interference between the scattered and the incident wave at the image point is usually analysed in terms of the phase contrast theoretical approach, which is employed for interpretation of HRTEM images. ${ }^{85-89}$ As a result of interaction with the specimen, the incoming electron wave is modified into a transmitted electron wave whose function $\psi(\mathrm{x}, \mathrm{y})$ contains information (the electron wave exiting from the specimen can be approximated as $\psi(\mathrm{x}, \mathrm{y})$ 
$=\exp \left[i \sigma V_{p}(x, y)\right]$, being $V_{p}(x, y)$ the electrostatic potential of the specimen projected in the $\mathrm{x}, \mathrm{y}$ plane and $\sigma$ an interaction constant $)^{85-89}$ on the structural details of the sample. Extraction of such information by formation of an image at the detector requires further interaction of the transmitted beam with the series of lenses constituting the microscope optics, in such a way that the observed image intensity distribution $\left(\mathrm{I}(\mathrm{x}, \mathrm{y})=\left|\psi_{\mathrm{m}}(\mathrm{x}, \mathrm{y})\right|^{2}\right)$ is correlated with $\psi(\mathrm{x}, \mathrm{y})$ through a function $\mathrm{T}$ that contains instrumental parameters (like apertures, wave attenuations, defocus and spherical aberration coefficient of the objective lens, etc.), according to ${ }^{85-89}$

$$
\hat{\psi}_{\mathrm{m}}(\mathrm{u}, \mathrm{v})=\hat{\psi}(\mathrm{u}, \mathrm{v}) \mathrm{T}(\mathrm{u}, \mathrm{v})
$$

where $\hat{\psi}_{\mathrm{m}}(\mathrm{u}, \mathrm{v})$ represents the Fourier transform of the wave function after the lenses, $\hat{\psi}(\mathrm{u}, \mathrm{v})$ is the Fourier transform of $\psi(\mathrm{x}, \mathrm{y})$ and $\mathrm{u}, \mathrm{v}$ are coordinates of the reciprocal space vector.

To take into account the influence of the different parameters affecting the contrasts observed in the experimental images (electron scattering by the sample particularly influences of irregular, columnar, etc. disposal of the scatterers -, lens imperfections, microscope instability, etc.), HRTEM quantitative analysis is usually based on matching experimental and calculated images, for which various computational methods have been developed. ${ }^{85,87,91,92,98,99}$ Figure 7 shows an example of application of simulated images to resolve geometrical aspects in a supported oxide system. ${ }^{91}$

Resolution limits of the TEM technique and schemes to achieve sub-angstrom resolution have been analyzed in detail by Spence. ${ }^{87}$ The highest structural resolution possible (point resolution) when the obtained images can be directly related to the projected structure of the specimen is given by the Scherzer expression $R_{s}=0.66 \lambda^{3 / 4}$ 
$\mathrm{C}_{\mathrm{s}}{ }^{1 / 4}$, where $\lambda$ is the electron wavelength and $\mathrm{C}_{\mathrm{s}}$ is the spherical aberration coefficient. ${ }^{87}$ From this expression and considering the de Broglie formula, higher resolution is achieved upon use of high-voltage instruments (acceleration voltages higher than 0.5 $\mathrm{MeV})$. Enhanced radiation damage, which may have stronger effects for nanostructured materials ${ }^{89}$ must however be considered in that case. Aberration correction has in turn allowed to achieve sub-angstrom resolution with microscopes operating at lower voltages (typically $200 \mathrm{KeV}$ ), allowing to resolve oxygen atoms in oxides materials. ${ }^{87,91}$ On the other hand, considering that high-resolution is achieved in TEM as the result of electron wave interference among diffracted peaks, a limitation to structural resolution can also arise from decreasing the number of atoms in the nanoparticle. ${ }^{99}$ This also can pose limitations to routinely employed determination of particle size distributions, particularly in cases when contrast differences between various sample components are not clearly resolved, as may occur for supported catalysts. ${ }^{99}$ Considering that such estimations are both microscope and sample dependent, calculations are generally required to establish limitations in contrast resolution in each particular case..$^{99,100}$

As mentioned above, information on chemical and electronic characteristics of the nanocrystals at a high spatial resolution is also achievable with TEM instruments. The use of scanning transmission electron microscopy (STEM) in which a fine convergent electron probe is scanned over the sample (the resolution being related to the probe size that can be as small as $\approx 0.1 \mathrm{~nm})^{93}$ is particularly useful for this purpose..$^{85,87,90,98,101}$ The electrons kinetic energy losses or the X-rays emitted as a consequence of inelastic scattering processes during electron-specimen interaction can be analysed by EELS and EDS techniques, which are the two most commonly used in chemical microanalysis. Due 
to EDS limitations in detection of light elements, EELS is most employed for the analysis of oxide materials. In addition to elemental information, the EELS spectra provide information on the electronic structure, bonding and nearest neighbor distribution of the specimen atoms. Among the different loss regions, the one most useful for this purpose is the high-loss one, related to electrons that have interacted with inner-shell or core electrons of the specimen atoms. Thus, the information obtained is similar to that given by XAS (see above), i.e. K-, L-, M-, etc. ionization edges of the elements present in the sample appear in the EELS spectrum (near edge and extended energy-loss fine structure ELNES and EXELFS - regions being defined within them). A generally higher energy resolution is however achieved with EELS, which comparatively facilitates analysis of the states present in a determinate band. ${ }^{89}$ An interesting example of the high spatial resolution that can be achieved with this type of instruments is provided by a recent TEM study of ceria nanoparticles (3-20 nm in diameter) in which EELS spectra are able to resolve between valence states of cerium at the edge in comparison to the center of the particles. ${ }^{102}$ Thus, even though as shown in a joint XPS-XANES study this phenomenon is still subject to some debate, ${ }^{103}$ such TEM-EELS study nicely show the valence reduction of cerium with decreasing the particle size and the preferential reduction of cerium at the surface of the nanoparticles. ${ }^{102}$

In connection with this, TEM images can be also formed with electrons that have lost a specific energy with respect to a determinate energy threshold of the atomic inner shell, giving rise to energy-filtered TEM (EF-TEM). A chemical mapping at the nanoscale can be obtained in this mode, which allows to resolve the location of the corresponding elements in the samples, being most useful in cases when small contrast 
differences between different sample components are observed in the bright-field images; in addition, differences observed in the EELS spectra as a function of the valence state of a determinate element can be exploited by EF-TEM for getting valence state distributions across the specimen. ${ }^{89,97}$

On the other hand, collecting the electrons scattered at high angles in a STEM (Scanning Transmission Electron Microscopy) instrument (by means of a high-angle annular dark field detector - HAADF -) has proven most useful to obtain images (by the so called Z-contrast imaging) of small clusters in catalysts or even single (relatively heavy) atoms or point defects. ${ }^{93,94}$ Considering the Mott formula for the electron scattering factor of a single atom $\left(\mathrm{f}_{\mathrm{e}}(\mathrm{s})=\mathrm{e}\left[\mathrm{Z}-\mathrm{f}_{\mathrm{X}}(\mathrm{s})\right] / 16 \pi^{2} \varepsilon_{0} \mathrm{~s}^{2}\right.$, being $\mathrm{s}=\sin \theta / \lambda, \mathrm{f}_{\mathrm{x}}$ the $\mathrm{X}-$ ray scattering factor of the atom, $\theta$ the scattering angle and $\lambda$ the electron wavelength), it is inferred that the electrons collected at high angles give significant information directly related to the atomic number. Another application of high utility in the analysis of materials, and in which significant progress has been achieved during the last years, is insitu TEM. ${ }^{93,104,105}$ Recent advances in this field are collected in the review of Wang. ${ }^{93}$

\subsection{STM and AFM}

Since the 1980's, scanning tunneling microscopy (STM) and STM-derived local probe methods have experienced a remarkable development. The seminal ideas behind these microscopies can be traced back to the early 1970s with the invention of the topografiner. ${ }^{106,107}$ Ten years later, Binnig and Rohrer established the basic principles for STM. ${ }^{108,109}$ For this important contribution, they received the 1986 Nobel price in physics. In a scanning tunneling microscope, the sample surface is scanned with a sharp 
tip located at a distance of less than a few nanometers. ${ }^{110}$ The tip is mounted on a piezoelectric positioner. A voltage is applied between the sample and tip, generating a tunneling current that varies exponentially with separation. ${ }^{108,110}$ The tunneling current is then measured, and a feedback control system compares the actual tunneling current with a (user-adjustable) "set current". If the actual current is too large or too small, the feedback control system moves the tip back or forward (respectively). In a first approximation, the movement of the tip represents variations in the position of the atoms in the surface of the sample. The two-dimensional array of numbers representing the surface "height" at each point is recorded in a computer, displayed in the computer screen, and then stored on a magnetic or optical device for subsequent analysis. ${ }^{110}$

Several approaches have been proposed for the analysis of STM images. ${ }^{111,112,113,114,115,116}$ In general, there is a relationship between the STM image and geometric and electronic properties of the surface of a sample. A precise interpretation of the image at an atomic scale can be a problem ${ }^{109,115,116}$ because the image is not at all a steric representation of the surface but is a view of its electronic structure at the Fermi level energy. In most cases, it is therefore not possible to directly and simply relate the bumps and shapes of the STM image to the actual lateral positions of atoms. ${ }^{116}$ One must establish how the structural parameters affect the tunneling current and formation of the image. ${ }^{117,118}$ A good knowledge of the electronic structure of the surface and tip is a prerequisite for the calculation of the tunneling current. To obtain this electronic structure different quantum mechanical approaches can be used ranging from the extended Hückel method to calculations based on density functional theory. ${ }^{111,116}$ Various levels of approximation have been proposed for the calculation of the tunneling current. ${ }^{11-117}$ 
Many of the methods used to calculate the current rely on perturbation theory. ${ }^{119,120,121,122,123}$ Following this formalism, the current between two electrodes (represented by $\mu$ and $v$ ) can be described as ${ }^{110}$

$$
I=(2 \pi|e| / h) 3_{\mu, v}\left[f\left(E_{v}\right)-f\left(E_{\mu}\right)\right]\left|M_{\mu v}\right|^{2} \delta\left(E_{v}+|e| V-E_{\mu}\right)
$$

In this equation, $f\left(E_{v}\right)$ represents the Fermi-Dirac distribution function (the first factor in the summation reflects the fact that electrons must tunnel from an occupied electronic state to an unoccupied electronic state); $\mathrm{M}_{\mu v}$ represents the tunneling matrix element between state $\mu$ on the sample and state $v$ on the tip. The $\delta$-function term reflects energy conservation during the tunneling process. The calculation of $\mathrm{M}_{\mu v}$ is not trivial. ${ }^{110,111,116}$ In the early work of Tersoff and Hamann a s-wave was assumed for the tip ${ }^{112}$ but in subsequent studies tunneling matrix elements have been calculated with $\mathrm{p}$ and $\mathrm{d}$ states as tip orbitals. ${ }^{110}$ A single metal atom and clusters have been used to model the tip. ${ }^{119-123}$

When using perturbation theory, the tip and the surface are treated separately, neglecting any interaction between them. Technically, this is valid only in the limit of large tip to surface distances. Methods have been proposed for the calculation of the tunneling currents that take into account interactions between the tip and sample through a scattering theory formalism. ${ }^{124,125}$ In principle, the tunnel event is viewed as a scattering process: ${ }^{116}$ incoming electrons from the sample scatter from the tunnel junction and have a small probability to penetrate into the tip, and a large one to be reflected towards the sample. Multiple scattering events can affect the current. ${ }^{126,127}$ Tip-induced localized states and barrier resonances seem to play an important role in the conductance. ${ }^{128,129}$

Although it may not be easy to obtain structural parameters from STM images, this microscopy can be used in a straightforward way to study the shape or morphology 
of oxide nanostructures as a function of temperature and under reaction conditions. ${ }^{130,131,132,133,134,135}$ Figure 8 illustrates how useful this technique can be. ${ }^{136}$ Initially, Mo nanoparticles are present on a $\mathrm{Au}(111)$ substrate. There is Au-Mo intermixing and the nanoparticles are tri-dimensional. Upon oxidation and formation of $\mathrm{MoO}_{3}$, the STM image shows a radical change in the morphology of the system. There is no more Au-Mo intermixing and two-dimensional aggregates of $\mathrm{MoO}_{3}$ spread out over the $\mathrm{Au}(111)$ substrate. ${ }^{136}$

The dependence of STM images on applied bias can be utilized as the basis for a true atomic-resolution tunneling spectroscopy. ${ }^{133}$ The effects of the voltage on the tunneling current depend on the electronic properties of the sample and, thus, information can be obtained about local density of states and band gaps. ${ }^{137}$ Tunneling spectroscopy data can be compared directly with area-averaging probes of electronic structure such as photoemission and inverse photoemission spectroscopies. ${ }^{138,139}$ Since the tunneling data can be acquired with atomic resolution, one could be able to directly visualize the spatial distribution of individual electronic states. ${ }^{138,139}$

In atomic force microscopy (AFM), a tip is mounted on a cantilever. ${ }^{110,111}$ Forces exerted by the sample on the tip bend the cantilever, and this deflection is used in a feedback system in a manner analogous to the tunneling current in the STM. The forces relevant to AFM are of electromagnetic origin. ${ }^{140}$ In the absence of external fields, the dominant forces are van der Waals interactions, short-range repulsive interactions, adhesion and capillary forces. ${ }^{140}$ The interaction between the tip and sample can be operated on either the attractive or repulsive parts of the interatomic potential curve. Most experiments for contact AFM are performed on the steeply rising repulsive part of the 
potential curve. ${ }^{110}$ Operation in the attractive region is intrinsically more difficult because it involves weaker interactions and requires the measuring of both the force and force gradient. ${ }^{110}$ However, true atomic resolution is easier to achieve using attractive-mode contact AFM. ${ }^{110,111}$ Images obtained with repulsive-mode contact AFM may show atomic periodicity while no truly achieving atomic resolution.

In the last decade, atomic force microscopy have experienced a significant transformation by using a vibrating probe to explore the surface morphology. ${ }^{140}$ Dynamic or non-contact AFM methods are emerging as powerful and versatile techniques for atomic and nanometer-scale characterization. ${ }^{110,111,140}$ In dynamic AFM, there are two major modes of operation: ${ }^{141,142,143}$ amplitude modulation and frequency modulation (AM-AFM and FM-AFM, respectively). Using FM-AFM, images with very good atomic resolution have been obtained for semiconductors and insulator samples. ${ }^{144,145,146}$ Because AFM does not involve the induction of a tunneling current between the probing tip and sample, it can be applied to study oxide systems that have a low conductivity (insulators or wide band-gap semiconductors). ${ }^{147,148}$

To obtain structural parameters from AFM images one must model the movement of the cantilever over the sample in an accurate way. ${ }^{110,140}$ To gain insight into the tip motion, several authors have considered the cantilever-tip ensemble as a point-mass spring, and thus the movement can be described by a non-linear, second-order differential equation: $^{: 49,150,151}$

$$
\mathrm{mI}+\mathrm{kI}+\left(\mathrm{m} \omega_{\mathrm{o}} / \mathrm{Q}\right) \dot{\mathrm{I}}=\mathrm{F}_{\mathrm{ts}}+\mathrm{F}_{\mathrm{o}} \cos (\omega \mathrm{t})
$$

where $F_{o}$ and $\omega$ are the amplitude and angular frequency of the driving force. $\mathrm{Q}, \omega_{\mathrm{o}}$ and $\mathrm{k}$ are the quality factor, angular resonance frequency and the force constant of the free 
cantilever, respectively. $F_{t s}$ contains the tip-surface interaction forces. In the absence of tip-surface forces, equation (5.6.2) describes the motion of a forced harmonic oscillator with damping. ${ }^{140}$ The mathematical expressions for $\mathrm{F}_{\mathrm{ts}}$ in general can be complex and contain terms for long-range attractive interactions, short-range repulsive interactions, adhesion, and capillary forces. ${ }^{140}$

\section{Acknowlegment}

J.A.R. and J.H. are grateful for the financial support of the US Department of Energy,

Division of Chemical Sciences (DE-AC02-98CH10886 contract). M.F.G. and A.M.A. thank the Spanish "Ministerio de Ciencia y Tecnología" and CSIC for financial support.

(1) Stout, G.; Jensen, L. X-ray Structure Determination A Practical Guide; John Wiley \& Sons: New York, 1989.

(2) Fernández-García, M.; Martínez-Arias, A.; Hanson, J.C.; Rodriguez, J.A. Chem. Rev. 2004, 104, 4063.

(3) Defect and Microstructure Analysis by Diffraction; Snyder, R. L.; Fiala, J.; Bunge, H. J., Eds.; Oxford Univ. Press: New York, 1999.

(4) Egami, T.; Billinge, S. J. L. Underneath the Bragg Peaks Structural Analysis of Complex Materials; Elsevier Ltd: Oxford, 2003; Vol. 7.

(5) (a)Guinier, A., X-ray Diffraction in Crystals, Imperfect Crystals and Amorphous Bodies; Dover, 1994 (b) Deb; B; Biswas,T.; Sen, D.; Basumallick, A; Mazumder S.; J. Nanoparticle Research 2002, 4, 91. (c) Ohnuma, M.; Hono, K.; Onnodera, H.; Ohnuma, S.; Fujimori, H.; Pedersen, J. J. Appl. Phys.2000, 87, 817.

(6) Jenkins, R.; Snyder, R. L. Introduction to X-ray Powder Diffractometry; John Wiley \& Sons, Inc.: New York, 1996.

(7) Azaroff, L.V. Introduction to Solids ; McGraw-Hill: New York, 1960.

(8) Kim, J. Y.; Rodriguez, J. A.; Hanson, J. C.; Frenkel, A. I.; Lee, P. L. J. Am. Chem. Soc. 2003, 125, 10684.

(9) Warren, B. X-ray Scattering; Dover: New York, 1990.

(10) Toby, B. H.; Egami, T. Acta Cryst 1992, A48, 336.

(11) Proffen, T.; Billinge, S. J. L. J. Appl. Cryst. 1999, 32, 572.

(12) Mamontov, E.; Egami, T. J. Phys. and Chem. of Solids 2000, 61, 1345. 
(13) Chupas, P. A; Hanson, J. C.; Lee, P. L.; Rodriguez, J.A.; Iglesias-Juez, A.; Fernandez-Garcia, M; (in preparation)

(14) Mamontov, E; Brezny, R; Koranne, M.; Egami, T. J. Phys. Chem. B 2003, 107, 13007

(15) Petkov, V., Trikalitis,P.N., Bozin, E. S., Billinge, S. J.L., Vogt, T. and Kanatzidis,M.G. J. Am. Chem. Soc. 2002, 124, 10157.

(16) Petkov, V., Zavalij, P.J., Lutta, S., Whittingham, M.S., Parvanov, V., and Shastri, S., Phys. Rev. B., 2004, 085410.

(17) Bateshki, M., Hwang,S.-J., Park, D. H., Ren, Y. and Petkov, V. J. Phys. Chem. B 2004 108, 14956.

(18) Norby, P.; Hanson, J. Catalysis Today 1998, 39, 301.

(19) Grunwaldt, J.-D.; Clausen, B. S. Topics in Catalysis 2002, 18, 37.

(20) Walton, R. I.; O'Hare, D. Chemical Communications 2000, 2283.

(21) Rodriguez, J. A.; Hanson, J. C.; Kim, J.-Y.; Liu, G.; Iglesias-Juez, A.; FernandezGarcia, M. J. Phys. Chem. B 2003, 107, 3535.

(22) Chupas, P. J.; Qiu, X.; Hanson, J. C.; Lee, P. L.; Grey, C. P.; Billinge, S. J. L. J. Applied Cryst ,2003, 36, 1342.

(23) Fuggle, J.C.; Inglesfield, J.E. "Unoccupied Electronic States”. Springer-Verlag, Berlin, 1992.

(24) See “ $X$-ray Absorption”. D.C. Konnigsberger, R. Prins, Eds. Wiley, New York, 1987.

(25) See articles in Chem. Rev. 2001, 101.

(26) Rehr, J.J.; Albers, J.C.; Rev. Modern Phys. 2000, 72, 621.

(27) Egelhoff, W.G. Surf. Sci. Rep. 1987, 6, 253.

(28) Lytle, F.W. Synchrotron Rad. 1999, 6, 123.

(29) Levine, I.N. "Molecular Spectroscopy"; Wiley, New York, 1975.

(30) Aberg, T. Phys. Rev. 1967, 16, 14201.

(31) Muller, J.E.; Jepen, O.; Andersen, O.K.; Wilkins, J.W. Phys. Rev. Lett. 1978, 40, 720.

(32) Natoli, C.R.; Benfatto, M.; Doniach, S. Phys. Rev. B 1986, 34, 4682.

(33) Rehr, J.J.; Albers, R.C. Phys. Rev. B 1996, 41, 8139.

(34) Sayers, D.E.; Stern, E.A.; Lytle, F.W. Phys. Rev. Lett. 1971, 21, 1204.

(35) Poiarkova, A.V.; Rehr, J.J. Phys. Rev. B 1999, 59, 948.

(36) Bunker, G. Nucl. Instrum. Methods Phys. Res. 1983, 207, 437.

(37) L.X. Chen, T. Rajh, Z. Wang, M.C. Thurnauer, J. Phys. Chem. B 1997, 101, 10688

(38) K.L. Yeung, A.J. Maira, J. Stolz, E. Hung, N.K.-C. Hu, A.C. Wei, J. Soria, K.-J.

Chao, J. Phys. Chem. B 2002, 106, 4608.

(39) Luca, V.; Djajanti, S.; Howe, R.F. J. Phys. Chem. B 1998, 102, 10650.

(40) SW Han, HJ Hoo, SJ An, J Yoo, GC Yi, Appl. Phys. Lett. 2005, 85, 021917.

(41) Winterer, M.; Nitsche, R. ; Hahn, H. Nanost. Mat. 1997, 9, 397.

(42) GE Rush, AV Chadwick, I Kosacki, HU Anderson, J. Phys. Chem. B 200, 104, 9597.

(43) P. Nachimuthu, W.-C. Shih, R.-S. Liu, L.-Y. Jang, J.-M. Chem., J. Sol. State Chem. 2000, 149, 408. 
(44) Z. Zhu, X. Zhang, R.E. Benfield, Y. Ding, D. Gandjean, Z. Zhang, X. Xu, J. Phys. Chem. B 2002, 106, 4569.

(45) Y. Nagai, T.Yamamoto, T. Tanaka, S. Yoshida, T. Nonaka, T. Okamoto, A. Suda, M. Sugura, Catal. Today 2002, 74, 225.

(46) Xijuan, Y.; Pingbo, X.; Qingde, S. Phys. Chem. Chem. Phys. 2001, 3, 5266.

(47) K. Asuka, Y. Iwasawa, Chp. 7 in "X-ray absorption fine structure for catalysts and surfaces"; Iwasawa, Y. (Editor); World Scientific, Singapure, 1996.

(48) Wachs, I.E. Catal. Today. 2005, 100, 79.

(49) S. Lemaux, A. Bansaddik, A.M.J. van der Eerden, Bitter, J.H.; D.C. Koningsberger, J. Phys. Chem. B 2001, 105, 4810.

(50) H. Oshitake, T. Suguhara, T. Tatsumi, Phys. Chem. Chem. Phys. 2003, 5, 767.

(51) J. Rockenberger, L. Troger, A. Kornowski, T. Vossmeyer, A. Eychmuller, J.

Feldhaus, H.Weller, J. Phys. Chem. B 1997, 101, 2691.

(52) Clausen, B.S.; Norskov, J.K. Topics Catal. 2000, 10, 221.

(53) Jentys, A. Phys. Chem. Chem. Phys. 1999, 1, 4059 and references therein.

(54) Yang, P.; Cai, X.; Xie, X.; Xie, Y.; Hu, T.; Zhang, J.; Liu, T. J. Phys. Chem. B 2003, 107, 6511 .

(55) Fernández-García, M. Catal. Rev. Sci. Eng. 2002, 44, 59.

(56) Kizler, P. Phys. Lett. A 1992, 172, 66.

(57) Loudon, R. Adv. Phys. 2001, 50, 813.

(58) see, for instance, J.R. Ferraro and K. Nakamoto. "Introductory Raman

Spectroscopy", Academic Press, Inc. 1994.

(59) Wachs, I.E. in Handbook of Raman Spectroscopy, I.R. Lewis and H.G.M. Edwards (eds.). Marcel Dekker, Inc. P. 799. 2001.

(60) Richter, H.; Wang, Z.P., Ley, L. Sol. St. Commun. 1981, 39, 625.

(61) Parayantal, P.; Pollak, F.H. Phys. Rev. Lett. 1984, 52, 1822.

(62) Campbell, I.H.; Fauchet, P.M. Sol. St. Commun.1986, 58, 739.

(63) Spanier, J.E.; Robinson, R.D.; Zhang, F.; Chan, S.; Herman, I.P. Phys. Rev. B 2001, 64, 245407.

(64) Kelly, S.; Pollak, F.H.; Tomkiewicz, M. J. Phys. Chem. B 1997, 101, 2730.

(65) Fangxin, L.; Jinlong, Y.; Tianpeng, Z. Phys. Rev. B 1997, 55, 8847.

(66) Rajalakshmi, M.; Arora, A.K.; Bendre, B.S.; Mahamuni, S. J. Appl. Phys. 2000, 87, 2445.

(67) Zhang, W.F.; He, Y.L.; Zhang, M.S.; Yin, Z.; Chen, Q. J. Phys. D: Appl. Phys. 2000, 33, 912.

(68) Bersani, D.; Lottici, P.P.; Ding, X. Appl. Phys. Lett. 1998, 72, 73.

(69) Xu, J.F.; Ji, W.; Shen, Z.X.; Li, W.S.; Tang, S.H.; Ye, X.R.; Jia, D.Z.; Xin, X.Q. J. Raman Spect. 1999, 30, 413.

(70) Zuo, J.; Xu, C.; Hou, B.; Wang, C.; Xie, Y.; Qian, Y. J. Raman. Spect. 1996, 27, 921.

(71) Zuo, J.; Xu, C.; Liu, Y.; Qian, Y. Nanost. Mater. 1998, 10, 1331.

(72) Kim, Y.K.; Jang, H.M. Solid. State Commun. 2003, 127, 433.

(73) Zhen, Z.; Tan, S.; Zhang, S.; Wang, J.; Jin, S.; Zhang, Y.; Sekine, H. Jpn. J. Appl. Phys. 2000, 39, 6293. 
(74) Tsunekawa, S.; Ishikawa, K.; Li, Z.-Q.; Kawazoe, Y.; Kasuya, A. Phys. Rev. Lett. 2000, 85, 3440 .

(75) McBride, J.R.; Hass, K.C.; Poindexter, B.D.; Weber, W.H. J. Appl. Phys. 1994, 76, 2435.

(76) Hungría, A.B.; Martínez-Arias, A.; Fernández-García, M.; Iglesias-Juez, A.; Guerrero-Ruiz, A.; Calvino, J.J.; Conesa, J.C.; Soria, J. Chem. Mater. 2003, 15, 4309.

(77) Wang, X.; Rodriguez J.A.; Hanson, J.C.; Gamarra, D.; Martínez-Arias, A.;

Fernández-García, M. J. Phys. Chem. B 2005, 109, 19595.

(78) Weber, W.H.; Hass, K.C.; McBride, J.R. Phys. Rev. B 1993, 48, 178.

(79) Siu, G.G.; Stokes, M.J.; Liu, Y. Phys. Rev. B 1999, 59, 3173.

(80) Diéguez, A.; Romano-Rodríguez, A.; Morante, J.R.; Bârsan, N.; Weimar, U.; Göpel, W. Appl. Phys. Lett.1997, 71, 1957.

(81) Jouanne, M.; Morhange, J.F.; Kanehisa, M.A.; Haro-Poniatowski, E.; Fuentes, G.A.; Torres, E.; Hernández-Tellez, E. Phys. Rev. B 2001, 64, 155404.

(82) Hinrichs, K.; Schierhorn, A.; Haier, P.; Esser, N.; Richter, W.; Sahm, J. Phys. Rev.

Lett. 1997, 79, 1094.

(83) Glinka, Y.D.; Jaroniec, M. J. Phys. Chem. B 1997, 101, 8832.

(84) Glinka, Y.D.; Jaroniec, M. J. Appl. Phys. 1997, 82, 3499.

(85) Williams, D.B.; Carter, C.B. Transmission Electron Microscopy, Plenum Press, New York (1996).

(86) Reimer L. Transmission Electron Microscopy, 4th Edition, Springer Series in Optical Sciences (Vol. 36), Berlin (1997).

(87 Spence, J.C.H. Mater. Sci. Eng. R 1999, 26, 1.

(88) Yacamán, M.J.; Díaz, G.; Gómez, A. Catal. Today 1995, 23, 161.

(89) Wang, Z.L. J. Phys. Chem. B 2000, 104, 1153.

(90) Thomas, J.M.; Terasaki, O.; Gai, P.L.; Zhou, W.Z.; Gonzalez-Calbet, J. Acc. Chem.

Res. 2001, 34, 583.

(91) Bernal, S.; Baker, R.T.; Burrows, A.; Calvino, J.J.; Kiely, C.J.; López-Cartes, C.;

Pérez-Omil, J.A.; Rodríguez-Izquierdo, J.M. Surf. Interf. Anal. 2000, 29, 411.

(92) Yacamán, M.J.; Ascencio, J.A.; Liu, H.B.; Gardea-Torresdey, J. J. Vac. Sci.

Technol. B 2001, 19, 1091.

(93) Wang, Z.L. Adv. Mater. 2003, 15, 1497.

(94) Datye, A.K. J. Catal. 2003, 216, 144.

(95) Martínez-Arias, A.; Fernández-García, M.; Iglesias-Juez, A.; Anderson, J.A.; Conesa, J.C.; Soria, J. Appl. Catal. B 2000, 28, 29.

(96) Iglesias-Juez, A.; Hungría, A.B.; Martínez-Arias, A.; Fuerte, A. ; Fernández-García, M.; Anderson, J.A.; Conesa, J.C.; Soria, J. J. Catal. 2003, 217, 310.

(97) Yin, J.S.; Wang, Z.L. Phys. Rev. Lett. 1997, 79, 2570.

(98) O’Keefe, M.A.; Buseck, P.R.; Iijima, S. Nature 1978, 274, 322.

(99) Bernal, S.; Botana, F.J.; Calvino, J.J.; López-Cartes, C.; Pérez-Omil, J.A.;

Rodríguez-Izquierdo, J.M. Ultramicroscopy 1998, 72, 135.

(100) Klenov, D.; Kryukova, G.N.; Plyasova, L.M. J. Mater. Chem. 1998, 8, 1665.

(101) Sun, K.; Liu, J.; Browning, N.D. J. Catal. 2002, 205, 266.

(102) Wu, L.; Wiesmann, H.J.; Moodenbaugh, A.R.; Klie, R.F.; Zhu, Y.; Welch, D.O.;

Suenaga, M. Phys. Rev. B 2004, 69, 125415. 
(103) Zhang, F.; Wang, P.; Koberstein, J.; Khalid, S.; Chan, S.-W. Surf. Sci. 2004, 563, 74.

(104) Hansen, P.L.; Wagner, J.B.; Helveg, S.; Rostrup-Nielsen, J.R.; Clausen, B.S., Topsoe, H. Science 2002, 295, 2053.

(105) Gai, P.L. Topics Catal. 2002, 21, 161.

(106) Young, R.; Ward, J.; Scire, F. Review of Scientific Instruments, 1972, 43, 999.

(107) Young, R.; Ward, J.; Scire, F. Phys. Rev. Lett. 1971, 27, 922.

(108) Binnig, G.; Rohrer, H. Review of Modern Physics, 1987, 59, 615.

(109) Rohrer, H. Ultramicroscopy 1992, 42-44, 1.

(110) Hamers, R.J. J. Phys. Chem. 1996, 100, 13103.

(111) Magonov, S.N.; Whangbo, M.-H. Surface Analysis with STM and AFM; VCH:

New York, 1996.

(112) Tersoff, J.; Hamann, D.R. Phys. Rev. B 1985, 31, 805.

(113) Hamers, R.J. J. Vac. Sci. Technol. B, 1988, 6, 1462.

(114) Feenstra, R.M. Phys. Rev. B, 1991, 44, 13791.

(115) Kubby, J.A.; Boland, J.J. Surf. Sci. Reports, 1996, 26, 61.

(116) Sautet, P. Chem. Rev. 1997, 97, 1097.

(117) Carlisle, C.I.; King, D.; Bocquet, M.-L.; Cerdá, J.; Sautet, P. Phys. Rev. Lett. 2000, 84, 3899.

(118) Golloway, H.; Sautet, P.; Salmeron, M. Phys. Rev. B, 1996, 54, R11 145.

(119) Tersoff, J.; Hamann, D.R. Phys. Rev. Lett. 1983, 50, 1998.

(120) Tersoff, J. Phys. Rev. B, 1989, 40, 11990.

(121) Chen, C.J. J. Vac. Sci. Technol. A, 1991, 9, 44.

(122) Tsukada, M.; Kobayashi, K.; Isshiki, N.; Kageshima, H.; Uchiyama, T.; Watanabe, S.; Schimizu, T. Surf. Sci. Rep. 1991, 13, 265.

(123) Tsukada, M.; Kageshima, H.; Isshiki, N.; Kobayashi, K. Surf. Sci.1992, 266, 253.

(124) Sacks, W.; Noguera, C. Ultramicroscopy, 1992, 42-44, 140.

(125) Tekman, E.; Ciraci, S. Phys. Rev. B 1989, 40, 10286.

(126) Sacks, W.; Noguera, C. J. Vac. Sci. Technol. B, 1991, 9, 488.

(127) Sacks, W.; Noguera, C. Phys. Rev. B, 1991, 43, 11612.

(128) Ciraci, S.; Baratoff, A.; Batra, I.P. Phys. Rev. B, 1990, 42, 7618.

(129) Kubby, J.A.; Wang, Y.R.; Greene, W.J. Phys. Rev. Lett. 1991, 65, 2165.

(130) Diebold, U. Surface Science Reports, 2003, 48, 53.

(131) Li, M.; Hebenstreit, W.; Gross, L.; Diebold, U.; Henderson, M.A.; Jennison, D.R.; Schultz, P.A.; Sears, M.P. Surf. Sci.1999, 437, 173.

(132) Surnev, S.; Kresse, G.; Sock, M.; Ramsey, M.G.; Netzer, F.P. Surf. Sci. 2001, 495, 91.

(133) Surnev, S.; Kresse, G.; Ramsey, M.G.; Netzer, F.P. Phys. Rev. Lett. 2001, 87, 086102.

(134) Norenberg, H.; Briggs, G.A.D. Phys. Rev. Lett. 1997, 79, 4222.

(135) Batzill, M.; Beck, D.E.; Koel, B.E. Phys. Rev. B, 2001, 64, 245402.

(136 ) Song, Z,; Cai, T.; Chang, Z.; Liu, G.; Rodriguez, J.A.; Hrbek, J. J. Am.

Chem. Soc. 2003, 125, 8060.

(137) Valden, M.; Lai, X.; Goodman, D.W. Science, 1998, 281, 1647.

(138) Hamers, R.J.; Tromp, R.M.; Demuth, J.E. Phys. Rev. Lett. 1986, 56, 1972. 
(139) Hamers, R.J. Annu. Rev. Phys. 1989, 40, 531.

(140) García, R.; Pérez, R. Surf. Sci. Reports, 2002, 47, 197.

(141) Martin, Y.; Williams, C.C.; Wickramasinghe, H.K. J. Appl. Phys. 1987, 61, 4723.

(142) Anselmetti, D. Lüthi, R.; Meyer, E.; Richmond, T.; Dreier, M.; Frommer, J.E.;

Güntherodt, H.-J. Nanotechnology, 1994, 5, 87.

(143) Albrecht, T.R.; Grütter, P.; Horne, D.; Rugar, D. J. Appl. Phys. 1991, 69, 668.

(144) Giessibl, F.J. Science, 1995, 267, 68.

(145) Sugawara, Y.; Othna, M.; Ueyama, H.; Morita, S. Science, 1995, 270, 1646.

(146) Kitamura, S.; Iwatsuki, M. Jpn. J. Appl. Phys. 1995, 35, L145.

(147) Smith, R.L.; Rohrer, G.S. J. Catal. 1996, 163, 12.

(148) Smith, R.L.; Rohner, G.S. MRS Symposium Proceedings, 1997, 497, 53.

(149) Chen, J.; Workman, R.K.; Sarid, D.; Hoper, R. Nanotechnology, 1994, 5, 199.

(150) García, R.; San Paulo, A. Phys. Rev. B, 1999, 60, 4961.

(151) Sahin, O.; Atalar, A. Appl. Phys. Lett. 2001, 78, 2973. 
Fig. 1a

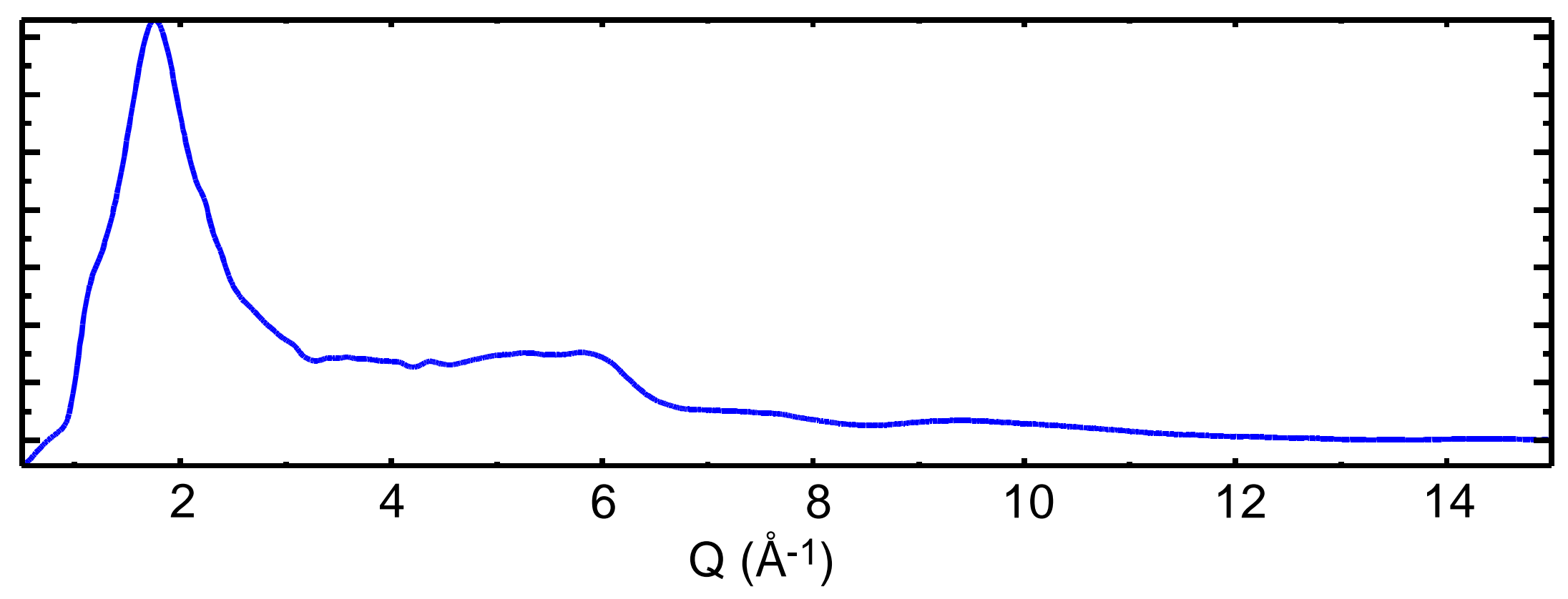


Fig. 1b
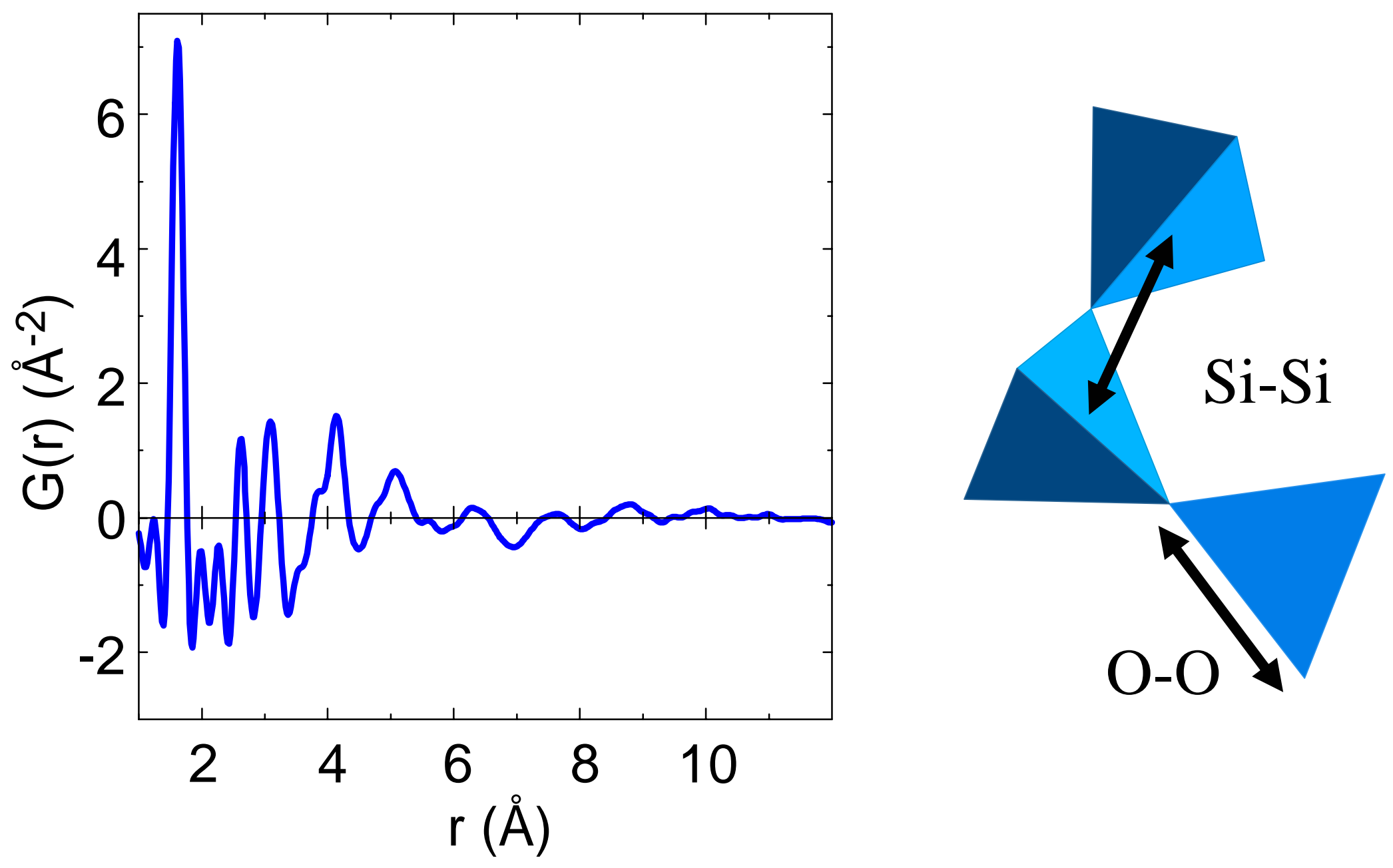
Fig. 2

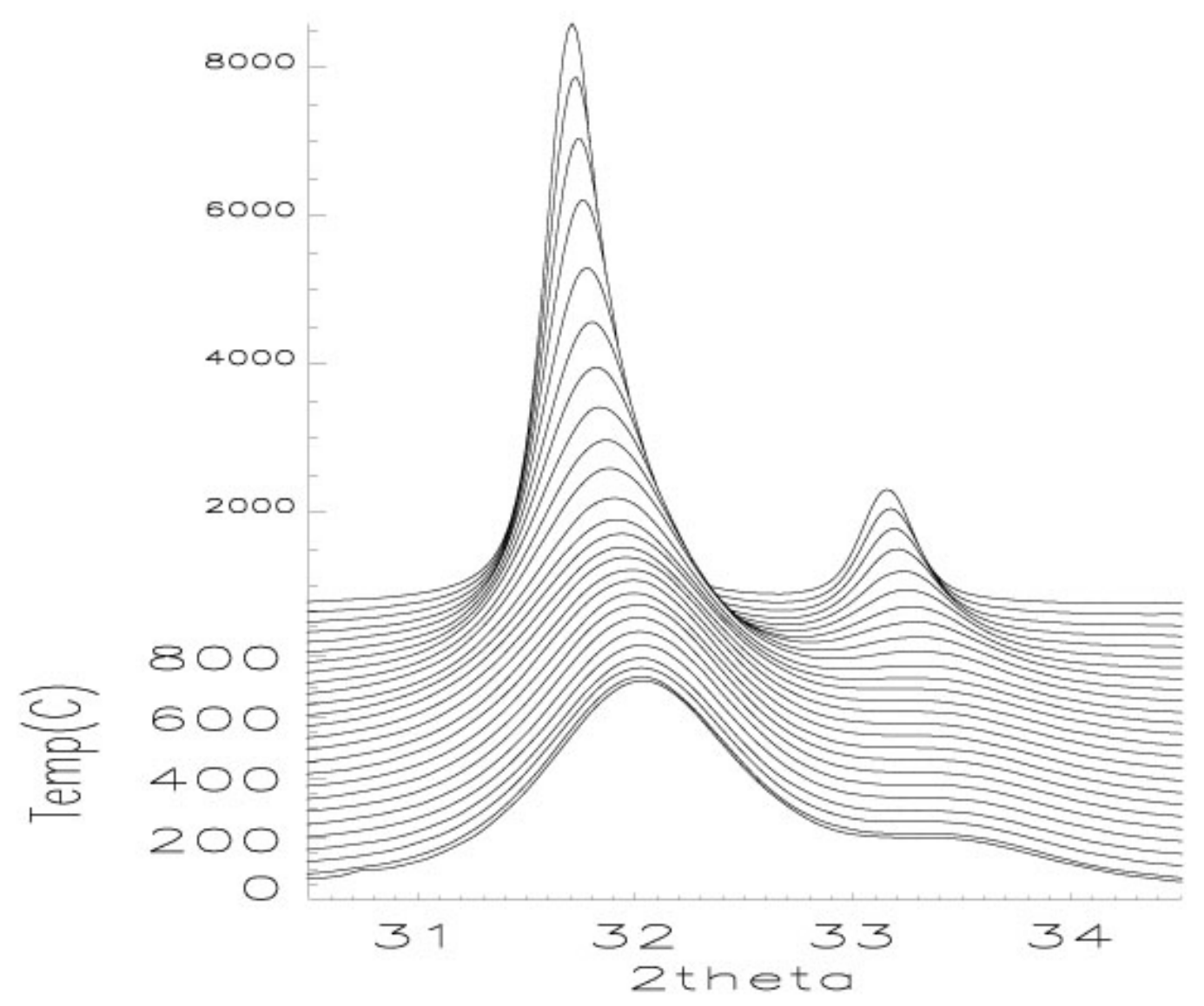


Fig. 3

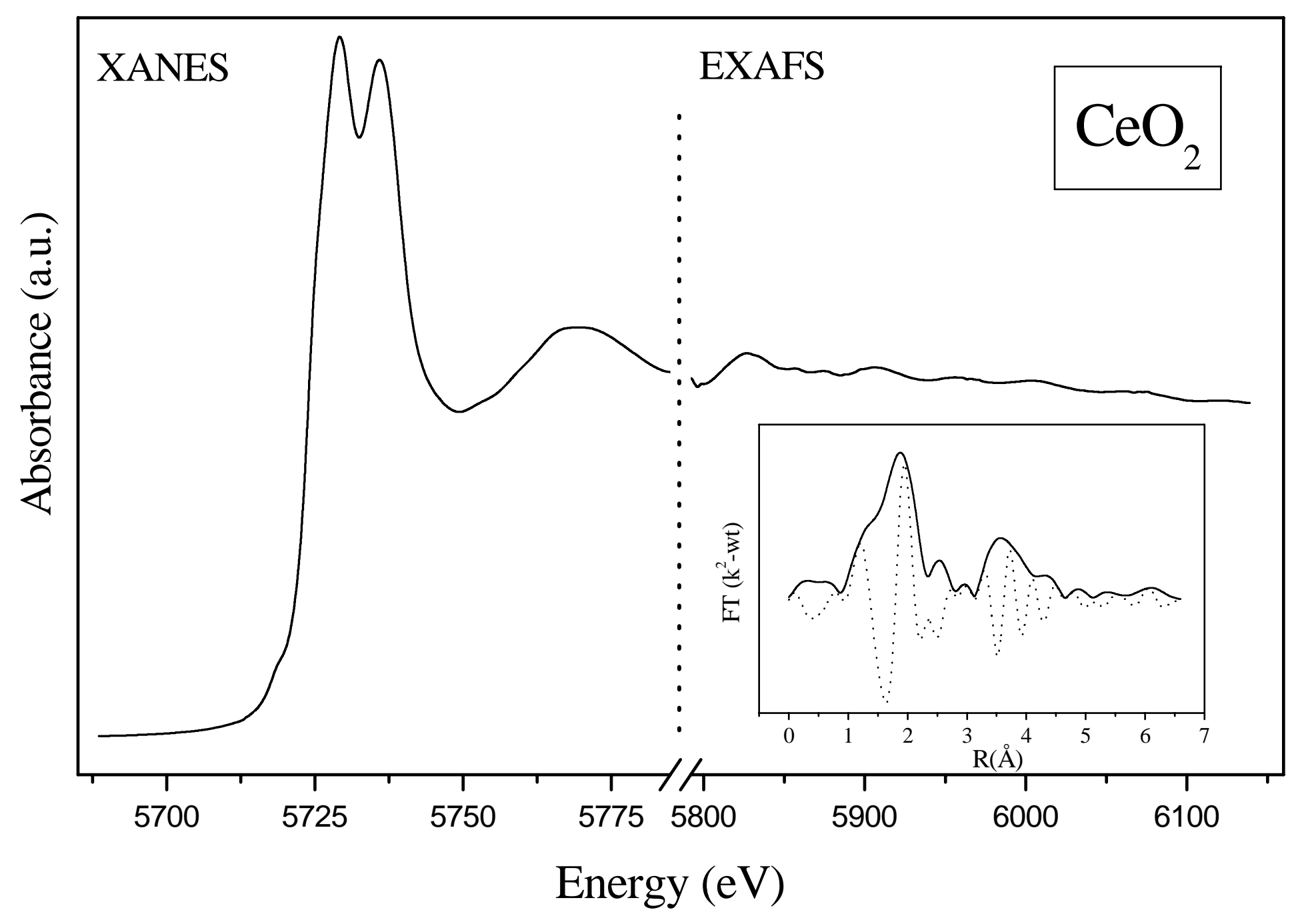


Fig. 4

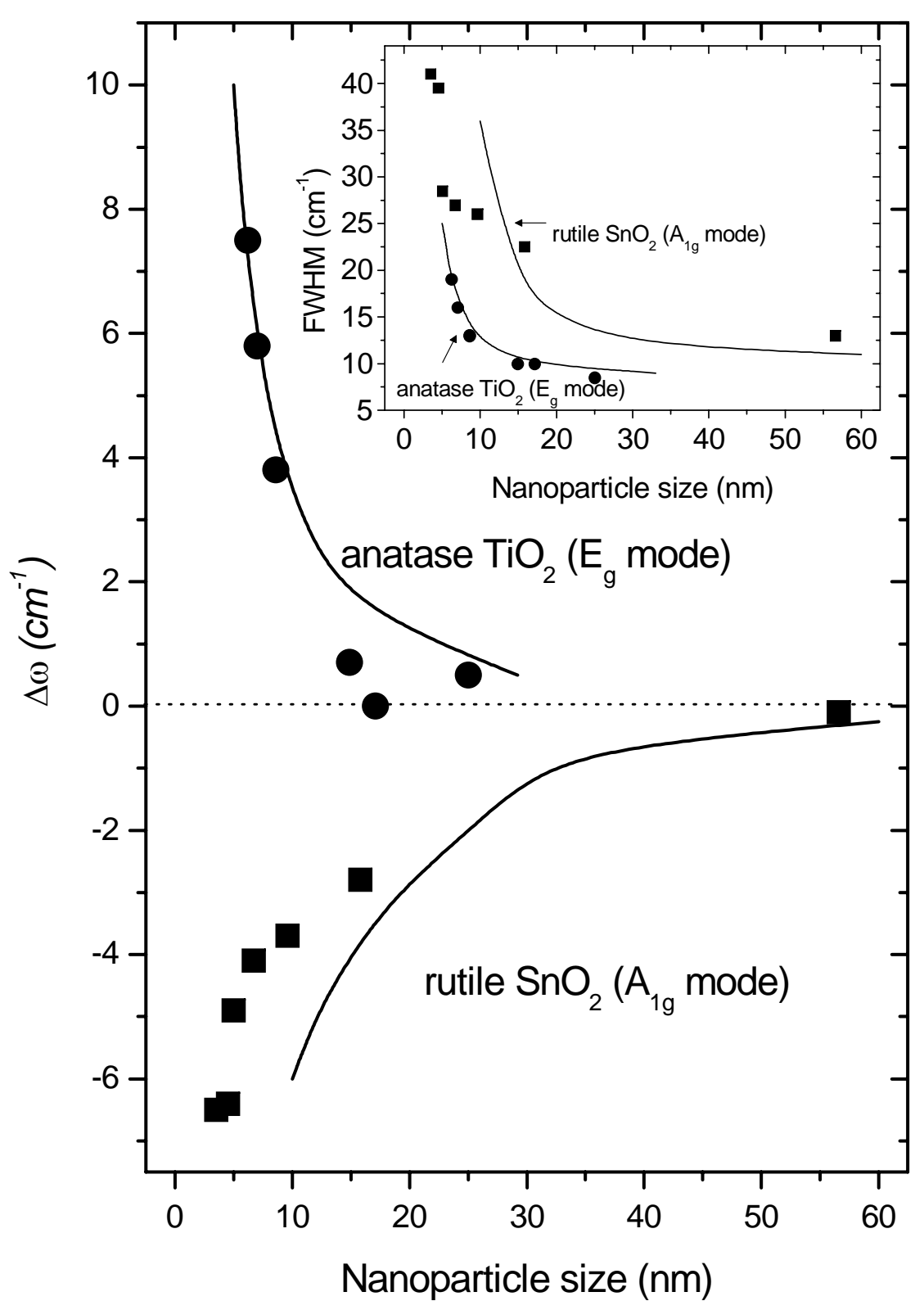


Fig. 5

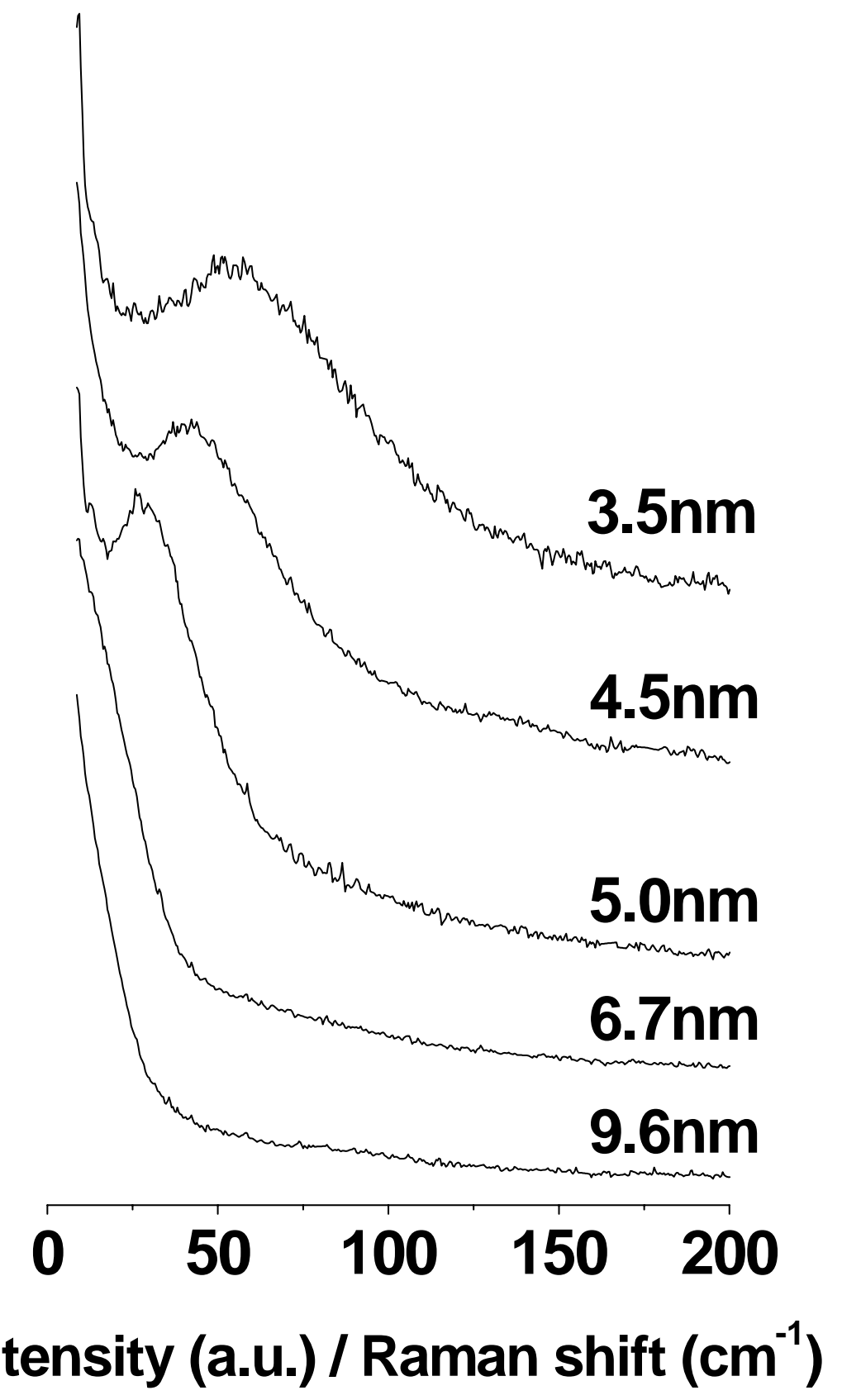


Fig. 6

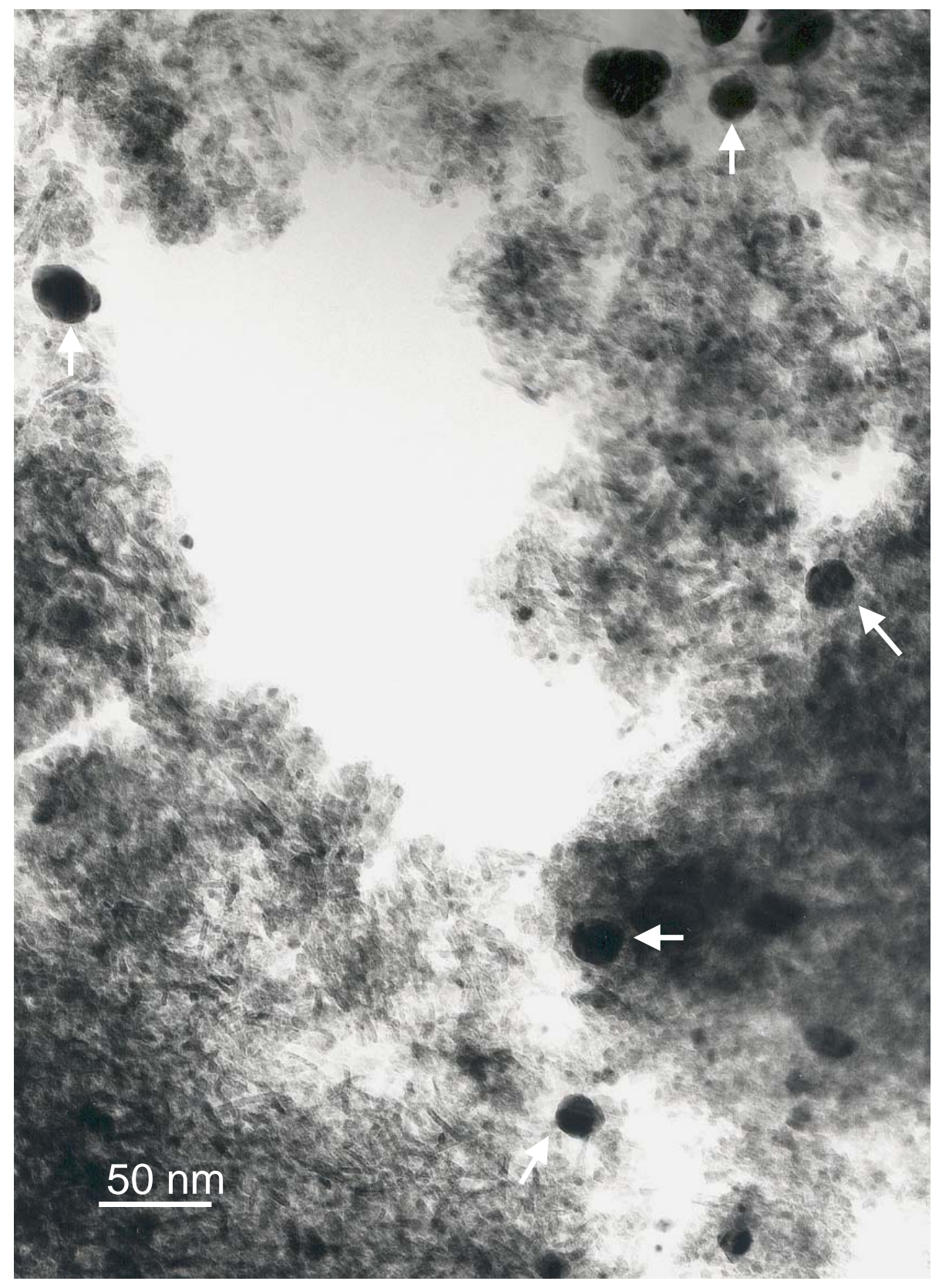


Fig. 7

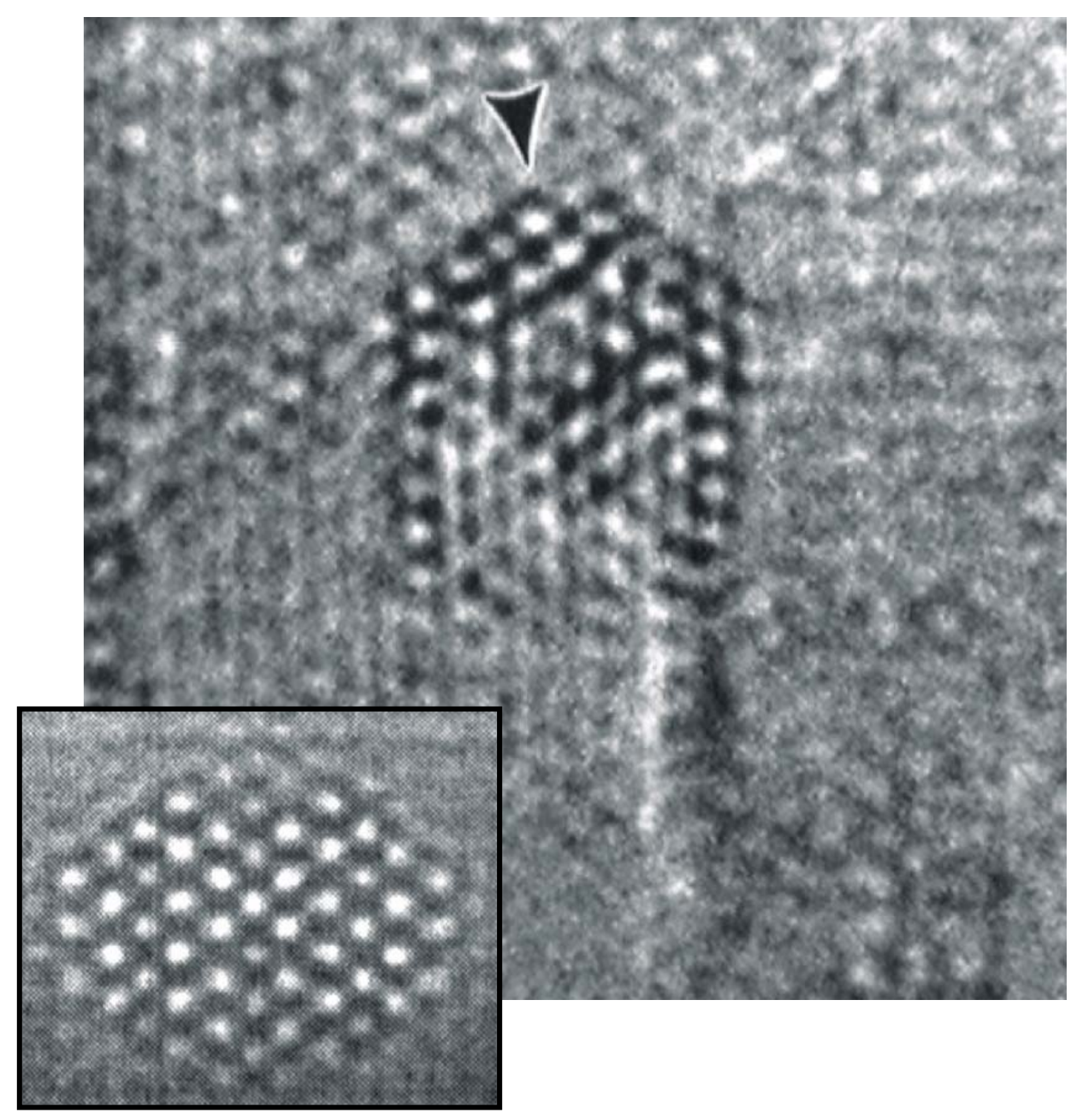


Fig. 8

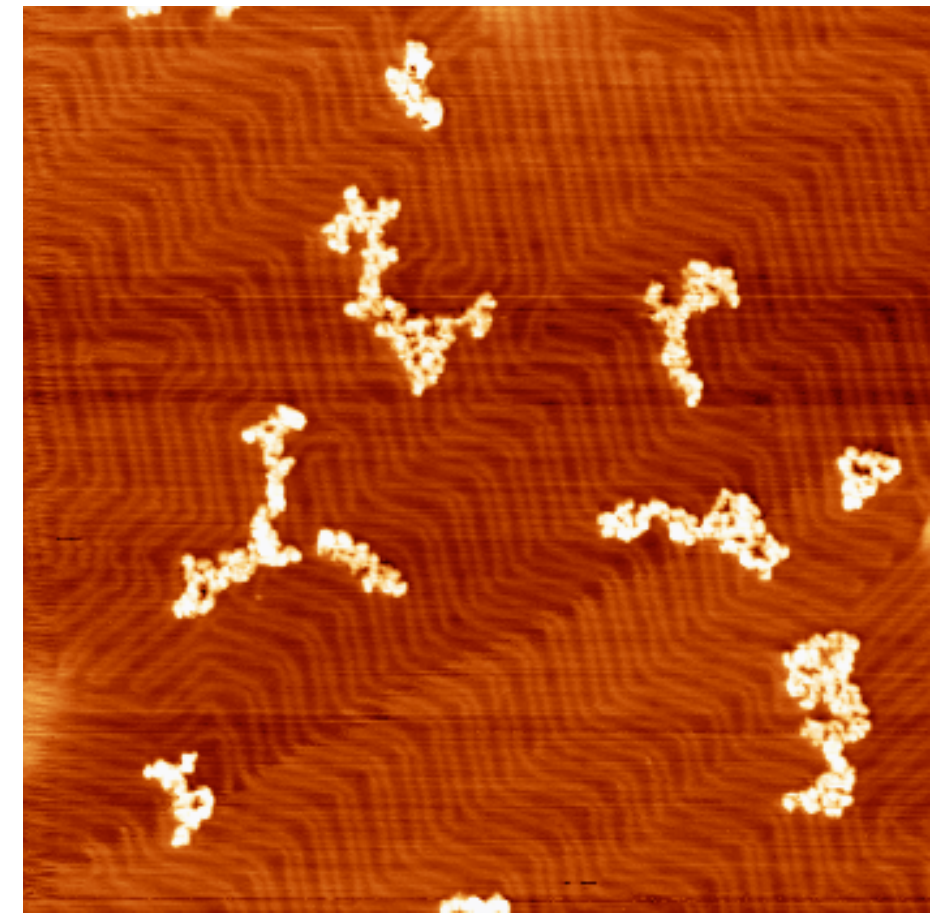

Mo/Au(111)

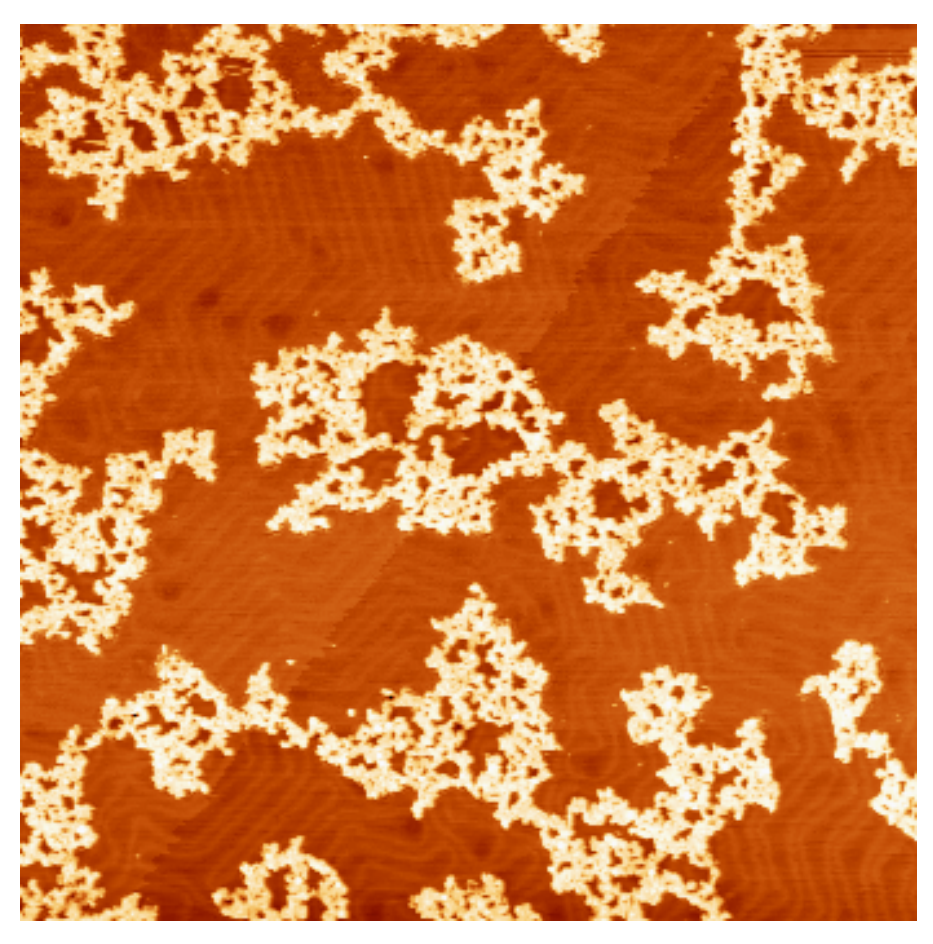

$\mathrm{MoO}_{3} / \mathrm{Au}(111)$ 